\title{
Graphene Membranes: Transport Properties and Energy Applications
}

\author{
Irma Rocio Vazquez and Sakineh Chabi* \\ Department of Mechanical Engineering, The University of New Mexico, Albuquerque, NM 87131, USA
}

*Corresponding author: Sakineh Chabi, Department of Mechanical Engineering, The University of New Mexico, Albuquerque, NM 87131, USA, Email id: schabi@unm.edu

\begin{abstract}
Graphene materials are increasingly being used in all types of membrane-based technologies ranging from water purification to energy conversion systems such as fuel cell and solar fuel technologies. Graphene's physical and chemical richness enables a variety of mass transport mechanisms that are impossible in traditional membranes. These mechanisms are dictated by the graphene microstructure and its versatility which leads transport to occur via pores, sub-nanometer pores, or via nanochannels. Graphene membranes can be designed to create nanochannels that allow highly selective ion or gas transport by biomimicking naturally occurring biological systems. However, these potentials cannot be fully explored without understanding the interplay between graphene microstructures and the transport mechanism. Toward taking advantage of graphene membrane technology, a fundamental understanding of the mass transport mechanisms is necessary. In this Review, we provide an in-depth discussion about three different types of mass transport through graphene nanopores and graphene nanochannels. By focusing on the relation between nanopores/channels chemistry e.g. effect of functional groups and pore polarity and transport mechanism, we identify key lessons, challenges, and potential solutions to empowering membrane-based energy technologies.
\end{abstract}

\section{Introduction}

Membrane separation technology holds a key role across various research areas and disciplines ranging from water desalination and purification systems to fuel cell technologies. Basically, membrane is a thin material which separates mass by selective transportation of one species of a mixture through it, while retaining other species behind. This ability to regulate permeation, has made membrane technology appealing in different mass separation applications. However, throughout membrane technologies, there are hurdles to overcome to achieve an optimal membrane such as a tradeoff between permeability and selectivity, and a need for more robust materials [1-3]. In this regard, graphene membranes offer tremendous potentials for use in membrane technologies. Graphene membrane is only one or few atoms thick, but it possesses excellent mechanical, chemical and thermal properties, making it an ideal candidate for use as a membrane. These characteristics are very important for efficient mass transport. Unlike pure graphene, which is impermeable, graphene membranes contain nanopores or nano channels which allow selective transport of mass, such as ions and gases. Mass transport through graphene can be classified into two groups: (i) transport via nanopores graphene (NPG) and (ii) transport via nano channels (NCs). Depending on the structure of the membranes (NPG, or NC), and their structural and chemical properties, there will be different resulting mechanisms and interplay occurring when mass transfuses through the membrane. These mechanisms will be discussed in detail in the next sections. As aforementioned, pure graphene is impermeable, thus pores need to be introduced to create a permeable material. The next section will discuss the main synthesis techniques used to create graphene membranes and highlight the main advantages and drawbacks of these approaches.

\section{Synthesis Approaches}

Graphene membranes can be prepared by a variety of methods. The effectiveness and applicability of each approach varies from one application to another, and it depends on many parameters including materials uses and capability, operation condition and synthesis cost.

\section{Nano Porous Graphene (NPG) Membranes}

A variety of techniques can be used to create nanopores in graphene. This includes methods such as drilling pores through a focused ion [4-10] or electron beam [11-13], etching [14-16] chemical activation [17-21] and electrical pulses [22]. Focused ion beam (FIB) drilling is one of the most commonly used techniques to create porous graphene, $[7,23]$ as it allows the formation of pores with broad diameters ranging from $10 \mathrm{~nm}$ to $1 \mu \mathrm{m}[7,15,24,25]$. 
Basically, in this method a beam of ions, such as Ga [4,6-9], Ar,[5] $\mathrm{He}[7,26]$ or Xe [10] ions, is used to bombard a selected graphene area $\left(\mathrm{nm}^{2}\right)$, sputtering carbon atoms from the surface and leaving holes behind. The shape, and dimensions of the created holes and pore density are affected by the ion diameter and ion type (larger dimeter creates larger pores), exposure time, and energy level of the ion beam (electron volt). Further, the capability to focus the ion beams on a nanometer dimension (slightly larger than the diameter of the ion beam) on graphene materials is another important factor. The electron irradiation technique is very similar to the FIB method except that, an electron beam instead of ion beam is used as the source of irradiation. The size and dimensions of created pores are determined by the intensity of electron beam and electrons properties $[12,26,27]$. In some scenarios, irradiation by electron beam or even ion beam generates relatively small pores or simply produces defects. In this cases, further treatment such as extra etching and high- temperature oxygen, hydrogen or oxidizing are required to produce more desirable pores [28].

For this reason, some research groups used a combination of methods to create a porous graphene material with desired pore structure and density. These include: ion beam bombardment followed by oxygen plasma etching [4] or ion bombardment followed by chemical etching e.g. acid etching [8] and combination of electron beam and ion beam [5], low-energy focused electron beam $(<10 \mathrm{keV})$ and nitrogen-ions [27] to induce high density pores in graphene membrane, or gain better distribution of pore size. Etching methods include plasma etching, where high speed plasma is shot in pulses at the graphene drilling high density nanopores on to the graphene membrane [14,15]. For example, R. Karnik's group reported the use of oxygen plasma etching to create pores in graphene for selective gas permeance [6]. Interestingly it has been found that by increasing the oxygen plasma exposure time, the treated graphene switches from a proton transport membrane to a proton and ion transport membrane, whereas for the argon plasma treatment, only protons are allowed to transport [15]. The oxygen plasma etching time not only increases the defect density but also enlarges the defects in graphene, whereas for argon plasma, extending the etching time only increases the density of the defects [15] Etching can also be achieved through $\mathrm{NaOH}$ solutions or via UVoxidative etching $[10,16]$. Another top-down approach to generate NPG membranes is chemical activation, which involves the use of various chemicals e.g., $\mathrm{KOH}$ to remove carbon atoms. This method is sought after for its capacity to produces graphene with highest specific surface area. For example, Zhang et al. [29] used a $\mathrm{KOH}$ assisted chemical activation process to create pores in graphene films. Doing so enabled the use of graphene for supercapacitors as pores are needed for charge storage [17].

\section{Graphene Oxide (G0) Membranes}

Contrary to the NPG membranes, GO-based membranes are mostly synthesized through bottom- up methods. The most common bottom method of GO membrane fabrication is vacuum filtration [20,29-45] During filtration, GO sheets are acted on by a gravitational or pressure force to stack one upon the other above a substrate which supports them. This allows the formation of nanocapillaries between the sheets and alongside them, which is what allows the transport of mass, such as ions and water or gas molecules. GO oxide can have an interlayer thickness of $\sim 0.6-1.2$ $\mathrm{nm}$ [38]. Different methods have also been successful in achieving a selective GO membrane such as drop casting GO sheets on different substrates such as polymeric, ceramic substrates or paper, [42,4648] or applying centrifugal force to deposit the sheets via a spin method on a ceramic substrate [42]. Methods such as spray-or spin-coating,[49] rod coating,[36] spray coating [36] and dippingrinsing [50] have not been explored as extensively.

\section{Graphene Frameworks Membranes}

Graphene framework membranes can include nano channels, 3D graphene, graphene/GO layered structure, GO composite and graphene nano meshes [51-54]. Graphene frameworks, unlike NPG and GO, can encompass a great variety of graphene-based membranes of different architectures, whether it be composed of oxide groups or be composites, etc. Top down approaches to produce graphene frameworks include methods such as block copolymer lithography,[51] nanoimprint lithography [52] and micromechanical cleavage.[53] Because of the variety of these structures, there is not one method that has proven to be the better one, but depending on the final desired structure, the synthesis can be adapted to fill the requirements. Graphene frameworks can also be designed using a variety of bottom-up methods. These can include sugar-blowing [55], spray coating [56], filtration. $[57,58]$ The synthesis method cannot be judged similarly to the NPG and GO ones, as the structures vary so much, but the overall goal is membrane selectivity, which has been achieved by both top-down and bottom-up approaches. Thus, indeed depending on the membrane types e.g., nanochannels or nano pores, different synthesis methods need to be adapted. Criteria of each fabrication method should be considered, such as the substrate when doing vacuum filtration and the type of ions when doing FIB drilling. The application should also be considered to best fit the membrane to have the best desired selectivity and best performance.

\section{Graphene Membrane Applications}

Owing to its excellent mechanical strength and flexibility, chemical modifiability and atomic thickness, graphene membrane can be considered as the ultimate solution for many energy related applications, ranging from water purification, and electrolyzer to fuel cells and supercapacitors. As such, it is essential to understand trends of recently studied graphene membrane systems and where the most efficiencies lie, both in experimental work and in theoretical calculations, for more comprehensive designs. In the next section we will review the main applications of graphene membranes.

\section{Energy Conversion and Storage}

A variety of electrochemical energy storage and conversion system such as fuel cells or supercapacitors use ion conductive membranes e.g., Nafion ${ }^{\circledR}$ or porous carbon for proton conduction 
or charge storage purposes, among other functions. As such, graphene membranes have great potentials for these systems. Common proton exchange membranes (PEMs) such as Nafio $n \AA$ are disadvantaged in many ways. At temperatures above $80{ }^{\circ} \mathrm{C}$, Nafio $n \circledast$ membranes start to lose water to evaporation. As a result, membranes lose proton conductivity due to poor mechanical stability [59-61] High temperatures are also known to cause a significant increase in fuel crossover. Hydrogen crossover is a major problem in both fuel cell and solar fuel technologies $[3,62]$. Increased levels of relative humidity (RH) is yet another substantial disadvantage of Nafio $n \AA$ membranes. Apart from Nafion $₫$ there are several other PEM materials available. For example, perfluorinated polymers are widely used in low temperature applications in fuel cells, for their high proton conductivity and stability [63]. However, perfluorinated polymers fail to address one of Nafion's major disadvantages of decreased proton conductivity at high temperatures. In addition to this limitation, perflourinated protonexchange polymers, are still liable to humidity and suffer from a thickness limitation $[53,64]$. In this regard, various recent research have demonstrated that graphene-based membranes are powerful alternative to Nafio $n \AA$ for their thermal stability, decreased susceptibility to humidity and gas crossover, and enhanced overall properties.[60,61,63,65-67] Graphene-based membranes such as graphene- Nafion ${ }^{\circledR}$ membranes or sulfonated poly(ether ether ketone) (PEEK) polyme- GO membranes demonstrated great proton conductivities and stability at elevated temperatures, over $100^{\circ} \mathrm{C}$, and increasing $\mathrm{RH}$. Or, graphene oxides provide interconnected channels along the surface of the graphene which is ideal for ionic transport over Nafion $®[61,65,68,69]$ At elevated temperatures, GO + Nafion ${ }^{\circledR}$ membranes are shown to retain more proton conductivity than Nafion $\AA$ alone. [59] Or, Zwitterion-coated GO (ZC-GO) membranes were reported to show better performance than Nafion ${ }^{\circledR}$ membrane. 25 wt\% ZC-GO membranes exhibited a high proton conductivity $\left(4.12 \times 10^{-2} \mathrm{~S} \mathrm{~cm}^{-1}\right.$ at $90{ }^{\circ} \mathrm{C}$ and $\left.\mathrm{RH} 100 \%\right)$ and low methanol permeability $\left(1.38 \times 10-7 \mathrm{~cm}^{2} \mathrm{~s}^{-1}\right.$ at $\left.25^{\circ} \mathrm{C}\right)$, which is much lower than that of Nafion $\AA$ under the same conditions. This improved performance was attributed to the formation of protontransport channels in the membranes [70-75].

The mechanism of ion/proton transport through graphene membranes will be discussed in detail in the next sections. Basically, two of the major properties influencing the transport of ions across a PEM are ionic conductivity and water uptake (WU). Performance of PEMs is also influenced by the swelling ratio of the material being used. The higher the swelling ratio, the PEM performance becomes more inferior [60] WU and swelling ratio determine just how hydrophilic the membranes are. Water does act as the carrier that transports the proton ions through the walls of the membranes. Significantly elevated WU measurements lead to dimensional instability [76] For example Pure SPEEK demonstrated a high degree of dimensional swelling .However the use of graphenebased membrane can help reduce the swelling [69]. Further, the proton conductivity of sulfonated polymer PEMs is directly related to the degree of sulfonation of the polymers. Because membranes with high sulfonation tend to be very hydrophilic, this causes major swelling, poor mechanical properties, and fuel permeability, causing a disadvantage [61] However, graphene membranes such as GO with sulfonic acid groups paired with Nafion ${ }^{\circledR}$ improves the compatibility. Ultimately, this indicates that the incorporation of graphene into PEMs optimize the overall performance [77]. Recently, GN-based membranes have also been used successfully as anion exchange membranes (AEMs). For example, functionalized graphene nanoribbons have been used successfully as an AEM for alkaline fuel cell and other applications [70-75] The use of graphene-based anion exchange membranes improved the properties of membranes in the form of mechanical and thermal stability, hydroxide conductivity, overall antifouling performance [78-80]. Similar to fuel cell applications, graphene may be used for solar fuel technology, and water electrolysis $[3,81]$ Solar fuel and fuel cell technology are very similar and as such, graphene-based ion exchanges membranes can be applied in both. In the former solar energy is used to split water into hydrogen and oxygen and in the latter hydrogen and oxygen are combined to produce electricity.

Additionally, graphene membrane can be used to address gas crossover problem in solar fuel systems. Given that graphene membrane is impermeable to $H 2$ molecules but proton conductive, it can work perfectly for solar-driven water splitting systems. In addition to aforementioned advantages, the use of graphenebased materials such as graphene oxide or N-doped graphene may eliminate the need for catalyst for the redox reaction as graphenebased membrane can also serve as catalyst for water splitting, and oxygen evolution reactions [82-85]. Graphene membrane also holds great potential for battery and supercapacitor development. NPG is particularly attractive for supercapacitors as it has a very high surface area of about $2000 \mathrm{~m}^{2} \mathrm{~g}^{-1}$. The combination of pure capacitive behavior, high surface area and mechanical robustness can lead to excellent electrochemical performance for any supercapacitor. Such graphene-based supercapacitors benefit from both high energy density and high-power density. Additionally, NPGs have also been used successfully for batteries such as lithiumion batteries or lithium-sulfur batteries. For example, Luo et al. [86] reported an improved cyclic stability for Li ion battery by replacing silicon anode with silicon-graphene membrane. By embedding silicon nanoparticles in graphene pores, a free-standing flexible anode was created that were able to hold its initial capacity up to 1300 cycles at $400 \mathrm{~mA} \mathrm{~g}^{-1}$. Additionally, a variety of graphene membrane-based electrodes have been used recently as electrode materials for various batteries. Most of these new electrodes demonstrated much better mechanical ion transport, e.g., short fast ion pathways, and flexibility compared to graphene membrane-free electrodes $[87,88]$ High flexibility, improved power density, cyclic stability and self-discharging behavior have been reported for NPGs-based batteries. If pores can be created in a hierarchical order e.g. micro, nano, sub-nano, then excellent overall performance may be achieved [30,89-92]. Further discussion of GN-based batteries or supercapacitors is beyond the scope of this paper.

\section{Water Desalination}

The current crisis of water shortage in the world is gaining more attention. Lack of clean water brought upon by increased world population, pollution and industry consumption calls for attention 
and a solution for this problem [93] Efficient desalination methods are an ever-increasing need, [94] however, current desalination techniques are high energy alternatives (e.g. multistage flash, multiple-effect distillation and osmosis treatments) that lead to higher carbon footprints and costs. Reverse osmosis (RO) membrane technology is the most energy efficient desalination technique, with efficiencies of up to $1.8 \mathrm{kWh} / \mathrm{m}^{3}$ at a commercial plant [95]. Nonetheless current membrane technology suffers from the trade off in thickness and permeability. Recent insight for possible alternatives has exposed their disadvantages. In recent years, graphene has attracted great attention for use in water desalination. Graphene has properties that make it an ideal membrane candidate in a water purification application. Its great mechanical properties like yield modulus of $1 \mathrm{TPa}$ and intrinsic strength of $130 \mathrm{GPa}$, [96] make it the strongest material ever measured. This provides graphene with the potential of bringing mechanical strength to the water desalination system and withstanding high water flux. The monolayer thickness of graphene also introduces a critical element in efficient purification systems: permeation. Graphene does not have to compromise in this tradeoff, making it an excellent membrane option. Both porous graphene oxide membrane and nanochannels with a few- layer graphene has shown promise of high-water flux and high desalination efficiency. Recently, CohenTanugi and Grossman [97] reported a functionalized nano porous graphene membrane to have water permeability up to $66 \mathrm{~L} /\left(\mathrm{cm}^{2}\right.$ day $\mathrm{MPa}$ ) with over $99 \%$ salt rejection and water transport, one thousand times faster than conventional RO [98] Recently, graphene membranes have been able to have selectivity even of coions [99] Selectivity is critical as it leads to significant chemical, energy, and cost-efficient gains through better solute removal and thus more efficient processes [100].

\section{Other Applications}

Graphene has notable potential for applications in electronic systems, however its zero-bandgap inhibits its full capacity in electronics. [51,101] One solution to this problem is to use porous graphene such as graphene mesh for electronics devices. Various studies have shown that the creation of nanosized pores in graphene can open up a bandgap in graphene and turn semi-metallic graphene into a semiconductor. [51,52,102] Graphene membranes have also been used widely for gas separation. Gas separation is a critical process for many industrial and scientific processes such as natural gas sweetening, hydrogen separation from syngas, and carbon capture. A popular avenue for gas separation is via the use of membranes. $[6,7,41,103]$ However, these traditional membranes are limited in their overall selectivity/permeability because they suffer from a trade-off between selectivity and permeability. [104-106] Additionally, in the case of polymeric membranes, their structure will gradually change due to aging and plasticization, making a long-lived separation performance necessary for new membrane designs [107]. An ideal membrane should be as thin as possible to maximize flow rate, mechanically strong, and chemically inert to ensure its stability. Thus, owing to their atomic thickness, mechanical robustness and chemical properties, graphene membrane can be considered as the best candidate for gas separation process. Outside of energy applications, interest in graphene membranes has risen for its wide variety of application and uses including DNA sequencing and filtration. Further discussion of the application of graphene membranes is beyond the scope of this paper.

\section{Transport Properties of Nano Porous Graphene}

The overall performance of any electrochemical device is highly affected by its desired/unwanted mass transport process. For example, an ideal PEM fuel cell would benefit from fast proton transport, and minimum gas transport, and water flooding issues. As such, understanding the process of mass transport through graphene membranes is a key step towards achieving highly efficient graphene-based energy devices. Transport through nanoporous graphene, NPG, is highly affected by both graphene membrane microstructure and the transported molecules' properties. Depending on graphene characteristics e.g. pore distribution, chemistry of pores and the nature of interaction between graphene membrane surface and pores, and the transported species, e.g. ion or gas, different mechanisms govern the transport [108-111] Thus, understanding the relationship between processing-structure and transportation mechanisms in graphene membranes, and how these mechanisms are different than established mechanisms in traditional membranes, plays a key role in moving forward the graphene-based membrane technology. In this section, we will discuss three types of mass transport mechanisms: ion and proton transport, water transport, and gas transport.

\section{Ion Transport Mechanisms}

The transport mechanisms of ions are affected by three parameters: (i) the structure and chemistry of the pores e.g., pore size and density, functional groups, (ii) ion characteristics e.g. ion charge (cation or anion, divalent or monovalent) and ion size (e.g. hydrated ion radius), and (iii) the ion- pore interaction (e.g. steric exclusion, Donnan-type interactions, etc.), (Figure 1). This subsection will discuss in detail the different mechanisms that drive ion transport through NPG membranes and how there are usually multiple ones prescribing the transport. permeability. These characteristics, in turn, are affected by ion size, functional group, chemical structure, etc.

Steric exclusion: The steric exclusion mechanism is a size-based exclusion operation where the diameters of the graphene pores dictate the ions that will pass through, allowing smaller ions to permeate and blocking the larger ones. The main reason behind this exclusion is the diffusion energy barrier, which is the energy needed to overcome to pass through a pore. One of the most important criteria in this mechanism is ion dehydration. In the absence of external forces, such as pressure or an electric field, the water molecules encompassing an ion (hydration shell) prevent it from permeating an NPG membrane. Once such external forces are applied, which will be discussed in detail in upcoming sections, 
its hydration shell has to be reduced through steric exclusion. The ion, then, will detach from the water molecules in front of it when nearing the pore, and upon passage the new hydration shell will be established immediately [112] The dehydration of an ion decreases its barrier energy, allowing to more efficient ion transport through a pore. The hydration shell is so imperative that a larger ion with a hydration shell that is only partially shed permeates a graphene pore easier than a smaller ion with a more tightly bound shell $[108,113]$.

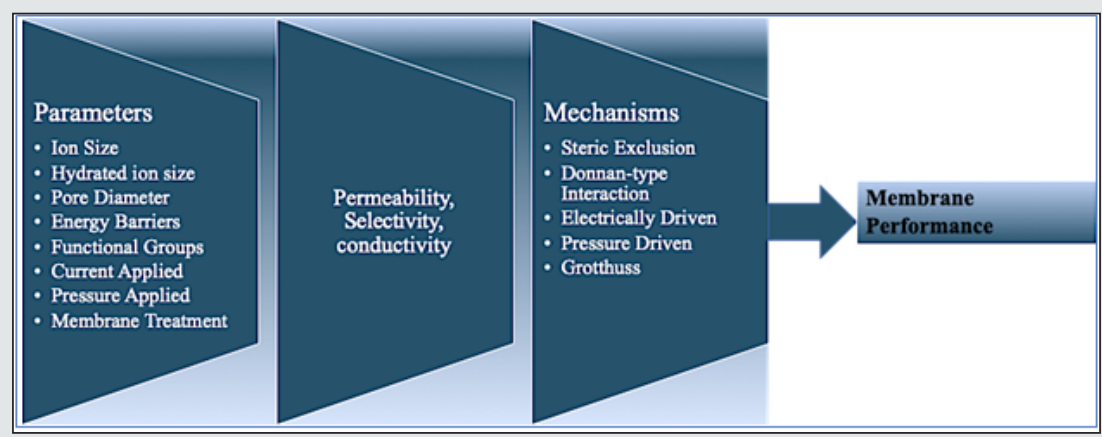

Figure 1: Relationships between graphene microstructure and ion transport mechanisms. The transport mechanism of ions through porous graphene membranes is determined by the membrane selectivity and permeability. These characteristics, in turn, are affected by ion size, functional group, chemical structure, etc.

The free-energy barrier depends on the ion valency, pore functionality and shape, and ion hydration structure [114,115] however in the absence of charges or added functional groups, the energy barrier is posed only by the dehydration [114]. One of the main challenges associated with steric exclusion is sieving molecules to similarly sized $\mathrm{H}_{2} \mathrm{O}$ molecules. For example, water and ethanol have a $0.3 \mathrm{~nm}$ and a $0.43 \mathrm{~nm}$ kinetic diameter, respectively, making a system that depends only on steric exclusion difficult. To efficiently separate these two, pore functionalization has been done such as adding $\mathrm{OH}$ [116] and $\mathrm{COOH}$ [117] groups, along with having an adequately sized pore. While not the sole selectivity mechanism in graphene membranes, steric exclusion, can be seen as a general trend where the NPG pore becomes bigger, the selectivity decreases [28]. It is of note to mention that in addition to large nanopores e.g. $20 \mathrm{~nm}$, sub nanometer pores and defects in the NPG membrane can also allow steric exclusion $[8,9,15,22]$ (Figure 2).

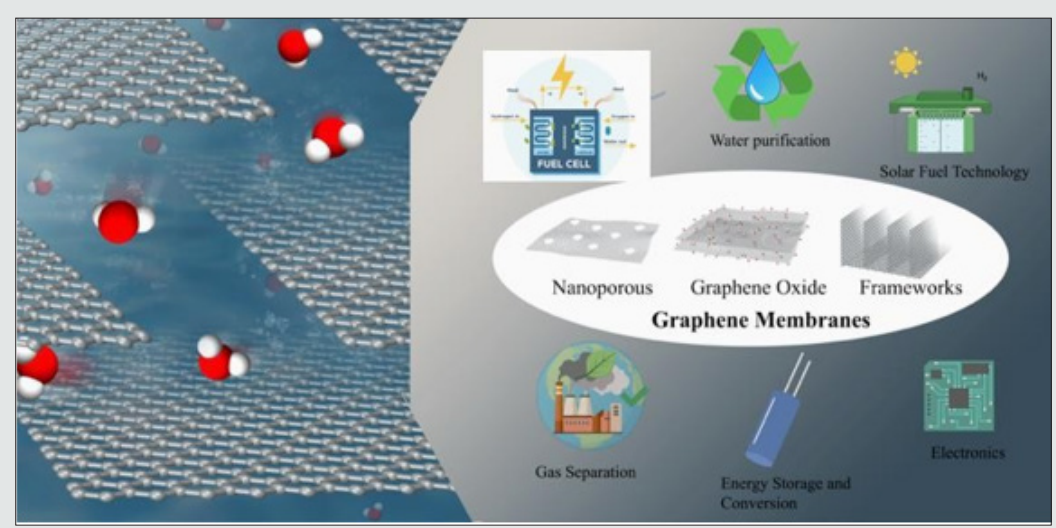

Figure 2: Graphene membranes applications in energy related technologies.

It is of note to emphasize that while most literature focuses on the ion size and pore chemistry to determine the transport mechanisms; recent studies strongly suggest that it is in fact the dehydration energy, or the size of the hydrated ions, that dictates the selectivity of the membranes. Thus, one potential approach to engineer the membrane selectivity is to facilitate the dehydration process of the targeted ions $[118,119]$.

Donnan Type and Other Related Mechanisms: Functionalized pores cause a transport mechanism that depends on the interactions of the ion and the atoms around a pore, often referred to as Donnantype transport mechanism. These interactions are characterized by pore functionalization and are not size dependent. To functionalize a pore, its edges can be passivated with ions, which induces specific electrostatic interactions and dictates the membrane performance $[44,120]$. Donnan-Type interactions depend mainly on the charge and the valence of charged pores. They cause coions (same charged ions) to repel each other and counter- ions (oppositely charged ions) to attract at the ion-membrane interface to maintain the electroneutrality of the ionic solution on both sides of the membrane [35,99]. For example, negative fluorine passivated pores can attract positive trihalomethanes through and result in high flux,[121] however hydrogen passivated pores 
can become hydrophobic, which prevents water from bonding with hydrogen, improving rejection, but in turn lowering the flux $[97,122]$ The selection of which functionalized charge groups the pores are passivated with is crucial in achieving a highly selective ion transport. Depending on what is required to be sieved through the graphene membrane, different passivation ions need to be selected. For example, an NPG membrane need to be functionalized with negative fluorine for cation selectivity, or positive hydrogen for anions selectivity [113]. Donnan-Type interactions are so significant that pores of up to $20 \mathrm{~nm}$ in diameter have shown $\mathrm{K}+$ / Cl- selectivity ratios over 100 , where the size of $\mathrm{K}^{+}$and $\mathrm{Cl}^{-}$ions are 0.133 and $0.181 \mathrm{~nm}$, respectively.[22] However, 100\% donnan-type transport cannot be realized in all systems and processes. Instead, there are usually multiple mechanisms at play during transport. For example a $\sim 5 \AA$ diameter negatively charged F-N pore allows $\mathrm{Li}+$, $\mathrm{Na}+$, and $\mathrm{K}+$ due to Donnan-type mechanisms, but it does so with ratios of 9:14:33, respectively, demonstrating the size selection of pores [113] Functionalized pores do not solely depend on the electrostatic interactions; the hydrophobic nature can also be exploited for selectivity. Hydroxyl groups (alternating $\mathrm{H}-, \mathrm{OH}-$ ) are hydrophilic and allow the water and hydrogen to bond, creating dipole interaction with water molecules [112] which is detrimental to ion rejection and doubles flux when compared to hydrogenated pores[97] or GO pores [122].

However, hydroxyl functionalized pores are effective at repelling $\mathrm{Cl}$ - even at moderate solution ionic strength [115]. This is likely because they yield high energy barriers for Cl- passage at low and moderate ionic strengths. Moreover, carbonyl functionalized pores (CO-pores) have shown $\mathrm{K}+$ selectivity over $\mathrm{Na}+$, as $\mathrm{Na}+$ has a higher energy barrier by $2.9 \mathrm{~kJ} / \mathrm{mol}$ [123]. It is also important to note that the carboxyl groups in the pores can strongly interact with salt ions much like $\mathrm{H}_{2} \mathrm{O}$ molecules, which results in a lower free energy barrier to ionic passing [122]. When the graphene sheet is functionalized with the COO- groups, it produces an effective energy barrier of $11 \mathrm{kcal} / \mathrm{mol}$ for $\mathrm{Cl}$ - ions as they attempt to permeate, thus having better rejection when compared to CNT pores [115]. Despite both - $\mathrm{COO}-$ and $\mathrm{NH}^{3+}$ pores having higher energy barriers for passage than pristine graphene because of electrostatic and steric effects, $\mathrm{NH}^{3+}$ proves to be less effective for repelling $\mathrm{Na}+$ and $\mathrm{Cl}$ - ions as the potential of mean force (PMF) is reduced at moderate ionic strength $(<4 \mathrm{kcal} / \mathrm{mol})$. A higher PMF denotes a higher barrier needed to overcome for ion passage [115] This leads $\mathrm{NH}^{3+}+$ functionalized pores accumulated with $\mathrm{Cl}$ - ion in the entrance, blocking even more Cl- ions[115]. Addition of oxygen atoms on NPG membranes has shown good selectivity as well. For example, It was reported that functionalizing graphene nano pores with oxygen atoms makes them exhibit a very good selectivity for $\mathrm{K}+$ over $\mathrm{Na}+(1000: 1)$, as $\mathrm{Na}+$ ions faces an energy barrier of about $21 \mathrm{~kJ} \mathrm{~mol}^{-1}$ higher than that for $\mathrm{K}+$ to pass through the nanopore. [124] In this case, both the size of ions and also the configuration of oxygen atoms ( $3.9 \AA$ Aistance between two oxygen atoms) play key roles in the transport process. It was discussed that since $\mathrm{K}+$ is larger than $\mathrm{Na}+$, it has better chances to stay in the center of the nanopores than smaller $\mathrm{Na}+$ ions. Thus, when a nanopore is efficiently sized, a balance between the electrostatic attraction and the repulsion from the van der Waals forces can be achieved for an ion in the pore center, which when the ions reaches the center of the pore it can prompt a continuous passage [124].

Indeed, Donnan-type interactions are affected by multiple parameters which require further exploration. For example, Donnan exclusion has been shown to weaken as electrolyte concentration increases [120] Low concentrations lead to a large Debye layer, which blocks the pores for co-ions, also leading to a high membrane potential [10], but ideal ionic concentrations are not known for ion selective transport through graphene membranes. Another parameter is electric field induced alongside pore passivation. For example, to maintain selectivity in a functionalized pore, an electric field can be introduced [113,123] but these are often not studied together. With proper coordination between pore size and passivation, Donnan-type interactions are excellent at bringing selectivity, and are effective in bringing behaviors like increased flux. However, controlling the surface charge, and sitespecific functionalization is still a challenge. Further, most of these studies are based on MD simulations and feasible methods to experimentally functionalize graphene membranes demands extensive investigation.

\section{Electric Field Assisted Ion Transport:}

Applying an external electric field on NPG membranes has proven to be effective in selectivity enhancement for ion separation. [113,123-128] Basically, the introduction of an electric field leads to selective ion transport due to a potential difference. When this occurs, an electric force is imposed on the ions, causing them to selectively permeate through the membrane [125] This mechanism has been studied for the separation of $\mathrm{Na}+$ and $\mathrm{Cl}-[125], \mathrm{K}+$ and $\mathrm{Cl}-[126,128]$ and $\mathrm{Na}+$ and $\mathrm{K}+[123,124]$ Lohrasebi et al.[125] reported that when the electric field magnitude is increased, the selectivity can be increased, however this usually needs to be fitted to each system's specific requirements [126] Effects of pore functionalization on electric-field assisted ion transport have also been studied. [123,124] For example, when the pores in He et al.[123] MD study were passivated with $3 \mathrm{COO}$ groups along the edges, the selectivity for either $\mathrm{Na}+$ or $\mathrm{K}+$ was controlled with changing the voltage: high voltages favored the passage of $\mathrm{K}+$, while lower voltages favored $\mathrm{Na}+$ passage. Interestingly, $\mathrm{K}+$ flux slows down because of its affinity to carboxylate groups. This continued interplay of various transport mechanisms is widely seen, where selectivity can depend on pore size, pore functionality and electric field that it is subjected to. In another example, Kang et al.[124] studied the use of electric field for ion transport through oxygen functionalized pores. They find that the electric field could boost membrane selectivity significantly providing that both the pore size and 0-0 distances are optimized, as the transport process is governed by the size of nanopores. It was also found that doping oxygen atoms into the graphene nanopores makes them exhibit a much better $\mathrm{K}+$ to $\mathrm{Na}+$ selectivity. Indeed, voltage dependent selectivity holds tremendous opportunity. However, this mechanism has mainly been investigated through simulation studies and still in 
its infancy and the difficulty of working with large area graphene poses a great challenge. Thus, it demands more attention, as introducing charge to NPG membranes, instead of depending on their steric properties opens the door to tunable selectivity.

\section{Pressure Driven Ion Transport:}

Another highly effective strategy in controlling ion transport through NPG is to build a pressure difference on either side of the membrane, driving the ions through it to bring equilibrium. The efficiency of pressure-driven transport is governed by the magnitude of the applied pressure, ion size/pore diameter, and pore functionalization[97] Additionally, it is affected by the interlayer distance in the case of multilayer graphene[58] One of the most important criteria in pressure-driven mechanism is the magnitude of applied pressure. In general, as the applied pressure for the transmission increases, so does the ion selectivity, however if it is too high, the system's energy efficiency drops. NPG membranes have demonstrated highly selectivity with pressures of up to $200 \mathrm{MPa}$. [121,129,130] However, realistic reverse osmosis pressures for water desalination are usually below 100 bar [131]. Thus, the general desired goal is the lowest pressure difference without sacrificing the membrane performance. For example, a successful account of this maintained $100 \% \mathrm{Na}+$ rejection with a $9.9 \AA$ Aiameter NPG but dropped the required applied pressure by $35 \%$ by adding functional groups to the pore edges [132] Studies of desalination with pressures ranges of 10-200 MPa have described the main driving mechanisms to be external applied pressure, even with functionalized hydrogen and hydroxyl pores [130].

Most of the reverse osmosis and forward osmosis (FO) studies have been achieved through simulations, but can a graphene membrane stand these pressure requirements experimentally? In an interesting study by L. Wang et al.,[133] it was found that wrinkles-free graphene can withstand up to 100 bars (10MPa) of pressure. it has been also reported that in an aqueous ionic solution, large ions are more susceptible to pressure changes than water molecules, [97] which is beneficial in bringing increasing ion rejection rates and increased water flux when the pressure increased in a system [28] This is something that can be exploited to create a high-water flux desalination system. Controlling the pore size is critical to achieve high selectivity in pressure driven systems. Cohen- Tanugi et al.[130] studied a reverse osmosis system where ion rejection decreased as the pore diameter increased. When the group optimized the pore size, they achieved $100 \%$ rejection which was also accompanied by high permeability (785.6 L per $\mathrm{m}^{2}$-h-bar) which is 3 orders of magnitude higher than the current RO permeance rates with commercially available resources. In another study, Azamat et al. [121] demonstrated full rejection of trihalomethanes (THMs) with a small pore size (6 $\AA$ diameter) at $200 \mathrm{MPa}$, however the water flow decreased after 100 MPa due to the blocking of the pores with THMs molecules. The optimization of each of the parameters is essential in obtaining a high-performance membrane. For RO studies the focus of studies has been on single layer, however bilayer graphene is a promising candidate in ion separation performance $[58,129]$ The interlayer distance of multilayer graphene can be as low as the $3.35 \AA$ (graphite interlayer spacing), and increased by setting barriers such as rigid nanoparticles like colloidal silica[58] between the layers. In an MD study an interlayer distance of $\mathrm{H}=8 \AA$, with a pore radius of radius $3 \AA$, demonstrated full salt rejection with pressures up to $200 \mathrm{MPa}$.[129] These multilayer systems do pose challenges, such as decreased water flow due to pore offset bringing a high transport barrier for water, and decreased rejection due to increased interlayer spacing and pressure. A solution to this would be balanced parameters such as small layer separation, and fully aligned pores.[129] As discussed earlier, there are indeed multiple mechanisms that drive ions through NPG membranes (Figure 3). Although various studies present steric exclusion as the main dominating ion transport mechanism through NPG membranes, not all ion transport is monopolized by this spatial operation, but rather by other behaviors or a combination of mechanism such as steric and Donnan-type, or steric and pressure driven mechanism.

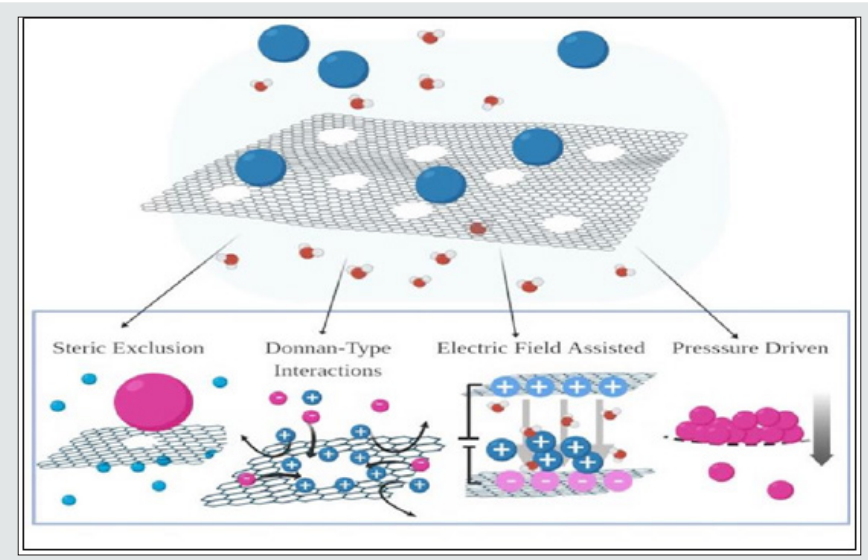

Figure 3: Ion Transport through Nanoporous Graphene (NPG) Membranes. The main mechanisms of ion transport through an NPG membrane: 1) Steric exclusion: where the selectivity is size based, 2) Donnan-type interactions: the electrostatic interactions between ions sieved and ion on the membrane surface, drive the selectivity, 3) Electric field assisted: the introduction of a voltage difference on the membranes causes the ions, based on their charge, to permeate to the other side of the membrane 4 ) Pressure driven: pressure driven the sieved ions through to the other side of the membrane. 


\section{Proton Transport}

Protons transport is a critical process in various electrochemical systems. For example, fast proton transport is a key step for water electrolysis, fuel cell, solar fuel, and proton battery devices. As discussed earlier, compared to traditional membranes such as Nafion ${ }^{\circledR}$, graphene membranes enable high proton conductivity as a result of short thickness, among other advantages. Compared with other ions, protons show distinct transport behaviors. One of the main reasons behind this distinction is the smallness feature of protons. For example, there is the possibility of proton and hydrogen passing through intrinsic defects within an NPG membrane with no need for external pores. [134-136] In important works by a group with A.K. Geim and others [53] it was reported that monolayers of pristine graphene are permeable to protons and can even have good selectivity for hydrogen isotopes.[137] Proton transport through graphene is complex depending on what the energy barrier from the characteristics of the membrane brings (e.g. pore size, existing functional groups, defects). Most of the proton transfer process can be explained through a Grotthus type mechanism, which consists of protons jumping from a molecule to another by breaking and forming $\mathrm{H}$ bonds simultaneously, this is especially true for graphene membranes with defects.[31,135] Selective graphene membranes have been fabricated, discriminating between proton vs deuterons,[136,137] protons vs helium and hydrogen,[135] and proton vs $\mathrm{H}_{2}$, water and methanol [53] Despite some superior proton transport, there are some challenges in pristine graphene films, such as achieving the optimal temperature for transport and the occurrence of blocked chemisorption due to hydrogenation, both of which largely affect the Arrhenius activation energy [136] Proton transport through NPG membranes has been shown to vary with defects whether they contain functional groups, their size and introduced voltage bias. The addition of functional groups to the defects has proven to be beneficial to proton transport. Achtyl et al.[135] reported that the hydroxyl terminated defects proved to be favorable for aqueous proton transfer, while Pyridium-like ether terminations stop proton exchange. Low-energy barriers for aqueous protons across $\mathrm{OH}$ - groups $(0.61-0.75 \mathrm{eV})$ on graphene defects made proton transport easier. The transport of aqueous proton through $\mathrm{OH}$-functionalized defects occur via Grotthuss mechanism.

It is of note to mention that defects play a key role on proton transport [15] Defects such as sp3 defects, can bring a lower required transmission energy for protons. The main restriction for proton transport across the graphene without defects is the energy required to pull the protons through the axis of a hexagon ring in the membrane. Additionally, proton transfer through graphene membranes depends on the physical and chemical properties of the membrane e.g. chemical composition of the membrane, whether it is pure graphene or graphene composites, as well as the number of graphene layers, and the size of the defects or pores $[53,136]$ These parameters that could also affect this are the application of an external electric field, functionalizing the pore or the defects, the size of the pore or the density of the pore. These mechanisms can bring great potentials for graphene as for example a proton exchange membrane, allowing it to be used for fuel cells and artificial photosynthesis.

\section{Water Transport}

The main characteristics defining water transport are pore size, water molecule structure, and external effects (e.g., charge and pressure), all of which are described below. Water molecules have a van der Waals diameter of $\sim 2.8 \AA$, so when the pores in NPG membranes are larger than $3 \AA$, water transport occurs without difficulty $[112,115,138]$ However, when the pore size is small, the $\mathrm{H}_{2} \mathrm{O}$ transport is influenced by hydrogen bonding as well as the water molecule structure, which is a product of the pore geometry and pore chemical functionalization.[9,112,115,131,138-141] Hydrogen bonding promotes the easiness of the water to go through a pore. Azamat et al. [121] reported that decreased hydrogen bonding is accompanied by decreased water transport through the pores. The structure of the water molecules changes when they pass through pores with diameters $<1.5 \mathrm{~nm}[115,140]$ Water molecules have the ability to adopt a quasi-vertical or flat orientation respective to the 2-D nanopore to facilitate the transfer through a porous membrane in the best energy-saving manner.[140] The structure of the water molecules near the pores is highly indicative of the pores' rejection capacity. In an MD simulation, water molecules that are free to move in 2D space had a decreased potential of mean force (PMF), allowing them to permeate through a pore easier than the rigid ones [115]. Water molecules find preferential positions within the pores and stay closer to the pore rim where they could interact with other water molecules $[112,115,140]$ Single file transport of water molecules has been seen in pores that can only fit a single water molecule in their cross section $[9,112,115,139]$ however when the pore size increases water flow becomes disordered, bringing inefficient use of the pore area [140]. Konatham et al. [115] argued that water flux was affected only minimally by the functionalization of the pores with either H-pores or F-pores because of the water molecules' electrical charge neutrality. Interestingly though, this is not always the case, as water was found to pass better though G-F pores than G-N pores.[138] The effect of the functionalization of pores is modest on water, yet water flow has been shown to increase by two-fold $[97,121,122,138]$ Depending on the functional groups, and whether they are hydrophilic or hydrophobic, the water flux can be decreased as well. [97] For instance, it was found that hydrophobic H-pores pores reduce the water flow by instituting conformational order. [97] Additionally, interesting trends have been seen in graphene membranes with F-pores that designate it an attractive prospect in applications with high power densities due to ultra-fast water transport. Terminating the F-pores has shown to bring stability to the graphene membranes,[112] resulting in F-pores showing higher water flux than GO membranes, or N-pores [138]. Pressure is another parameter that affects water transport. Applied pressure to water has been show to increase flow linearly $[131,139]$ and decrease the energy barriers of the water by causing the water molecules to move faster to the other side of the membrane [130]. 


\section{Gas Transport}

Gas transport through NPG membranes is affected by (i) the properties of graphene membrane e.g. membrane selectivity and permeability which in turn are determined by many parameters including pore size, and density, functional groups, and (ii) the molecular properties of the gases such as gas size and gas diffusivity
[104]. Steric exclusion is the central mechanism of selectivity for gas molecules through NPG. However, depending on the characteristics of the NPG and gas molecules, different mechanisms can dominate the transport process. The following sections will discuss gas transport through three main mechanisms: steric exclusion, Donnan-type mechanisms, and adsorption, (Figure 4).

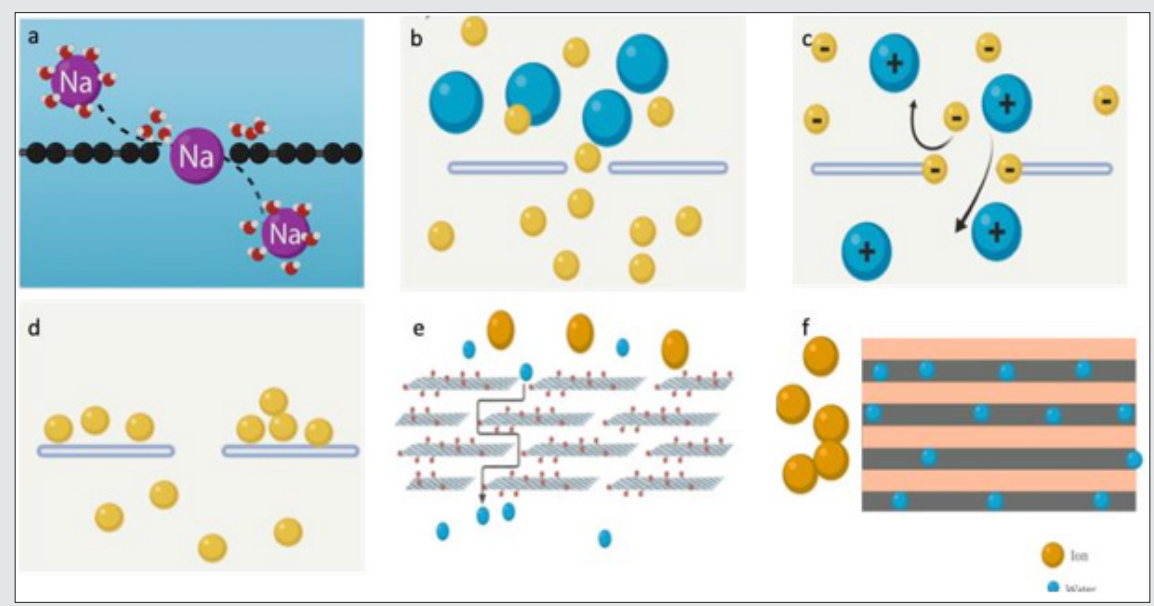

Figure 4: (a): ion dehydration process. Ion dehydration process plays a key role in the transport process, and it affects the permeation of ions into the membrane. regardless of the ion size, ions with smaller dehydration energies permeates easier than ions with large dehydration energy. (b-d) Gas Transport through NPG: b) Steric Exclusion, defined by spatial dependence c) Donnan-Type Interactions, defined by electrostatic (Coulombic) forces d) Adsorption, defined by weak noncovalent forces. (e-f) Ion transport through graphene nano channels. Ion transport through thenanocapillaries occurs parallel to the graphene plane.

\section{Steric Exclusion:}

The two most important parameters in gas steric exclusion through NPG membranes are the pore diameter and the gas molecules diameter.[108] Basically, steric exclusion on molecular sieving occurs when the gas molecule diameter is bigger than the pore diameter and it is impeded to go through the pore. This mechanism, similar to ion transport, can be affected by other parameters, such as the electrostatic interaction between the molecules and the pores, however the most defining characteristic is the pore size and kinetic radii of the target molecules. In a gas mixture, the varying molecules collide, and the transfer of linear momentum happens from lighter to heavier molecules, causing a collective flow, reducing the separation, so when a molecular mixture goes through graphene pores[7] the collision must be minimized or eliminated. Because of this, pores with diameters smaller than the mean free path are more efficient at separation. Ideally, the pore should be as small as possible, or just above the gas molecule size. For example, full separation of $\mathrm{H}_{2}$ from $\mathrm{N}_{2}$ was obtained through using a pore just bigger than the $\mathrm{H}_{2}$ molecule, but smaller than the $\mathrm{N}_{2}$ molecule.[107] Steric exclusion can be seen throughout the studies of gas permeation through NPG membranes. As mentioned, the pore size and the gas diameter are the most important parameters but can also be affected by other parameters such as the geometry of the pore $[107,142]$.

\section{Donnan-Type Interactions:}

Donnan-Type Interactions have a large impact on the selectivity of gas transport through NPG membranes. These electrostatic interactions during gas transport caused by either the added charges or functional groups attached to the pores of the graphene sheet, enhance the selectivity and prompt further investigation of the mechanisms of the interactions in molecular gas sieving. $[143,144]$ Functional groups can increase or decrease the diffusion energy barriers, which is the energy that needs to be surmounted so that the molecule permeates through due to the electrostatic or coulombic interactions,[143-145] which is why the atoms that are chosen for pore- passivation must be application specific and intelligently selected. For example, in the separation of $\mathrm{CO}_{2}$ and $\mathrm{N}_{2}$, the functional groups on the pore edges are what determine the selectivity. Graphene pores functionalized with fluorine [143] or nitrogen [146] show selectivity for $\mathrm{CO}_{2}$ but pores functionalized with hydrogen show selectivity for $\mathrm{N}_{2}[145,146]$ Interestingly, pores passivated with both nitrogen and hydrogen show selectivity for $\mathrm{CO}_{2} \cdot[144]$ The rationalization comes from the changes in the diffusion energy barriers. There is selectivity for $\mathrm{CO}_{2}$ in the N-pore due to the negatively charged $\mathrm{N}$ atoms which cause the $\mathrm{N}_{2}$ molecule to have a repulsive interactions at the pore [144] and because of the increase of electrostatic energy in the system inducing $\mathrm{CO}_{2}$ over $\mathrm{N}_{2}$ selectivity due to the quadrupole moment of $\mathrm{CO}_{2} \cdot[146]$ Additionally, 
upon $\mathrm{CO}_{2}$ passage, the molecules cause a sort of pulling interaction, increasing the $\mathrm{CO}_{2}$ uptake and blocking the pore for $\mathrm{N}_{2}$ permeation. [146] Contrary to the above results, a study with a hydrogen passivated pore (H-pore) shows selectivity for $\mathrm{N}_{2}$, allowing it to permeate with 105 GPU while having no CO2 permeation.[145] The reason for this selectivity is that $\mathrm{N}_{2}$ is almost electroneutral $\left(-0.061\right.$ e), whereas oxygen brings a more negative charge to $\mathrm{CO}_{2}$ $(-0.333$ e causes its atoms to have a strong electrostatic attraction resulting a higher diffusion energy for $\mathrm{CO}_{2}$ than $\mathrm{N}_{2}$.

Indeed, electrostatic interactions happening at the pore bring unique characteristics. Polar components are more likely to be affected by charges around the pore by strengthening their permeation. Such as in the case of the separation of $\mathrm{H}_{2} \mathrm{~S}$ and $\mathrm{CH}_{4}$, where $\mathrm{H}_{2} \mathrm{~S}$ greater polarity than $\mathrm{CH}_{4}$, causes it to accumulate around the pore, [105] where the stronger electrostatic interactions lower the potential energy of $\mathrm{H}_{2} \mathrm{~S}$ around the charged pore compared to that of the pristine pore, increasing the separation ratio. The passivation of functional groups can change the size of the pore, for example a common phenomenon seen in $\mathrm{H}$-pores is reduction of the pore size due to the $\mathrm{C}-\mathrm{H}$ bonds, leading to a decreased permeance when compared to a non-modified pore, which is important to keep in mind if attempting to design a high permeation pore.[143,146] In another similar study, Jiang's group [103] found that for $\mathrm{H}_{2} /$ $\mathrm{CH}_{4}$ separation, $\mathrm{N}$-pores demonstrated a high $\mathrm{H} 2$ permeance and selectivity on the order of 108 for $\mathrm{H}_{2} / \mathrm{CH}_{4}$. Whereas for an H-pore they found selectivity on the order of 1023, the difference being brought on by the Donnan-type interactions. The unmatched method for selectivity through Donnan-type interactions represents a sophisticated way to achieve a target gas separation ratio, however most of the studies for it are done through theoretical or simulations studies, creating a huge demand for experimental work for this method of selectivity.

\section{Adsorption}

An additional gas transport mechanism through NPG that often gets overlooked is adsorption. Adsorption refers to molecular gasses being attracted to the porous graphene surface through weak noncovalent forces such as van der Waals forces [146] These interactions are not as strong as electrostatic/coulombic forces (in Donnan-type interactions), however they are strong enough to maintain the molecules hovering close to the graphene sheet and leads to a different kind of permeation. The gas molecule diffuses to a pore and a potential well is created, followed by its passage the pore to the other side. As the adsorption height decreases (the molecule comes closer to the NPG), the interaction energy for the molecules reach into the attractive wells, thrusting the molecule through [145] . This is followed by the molecules dissociating from the area above the pore and desorbing from the surface. The entire process occurs in 5 steps:1) Adsorption 2) Association 3)Translocation 4) Dissociation 5) Desorption [147] . In this mechanism, the molecules form an adsorb layer of about $5 \AA$ thick from the graphene sheet, $[143,146,148,149]$ and strongly adsorbing gasses like $\mathrm{CO}_{2}$, can even have an adsorbed layer that is 3 to $4 \AA$ away from the graphene surface [149] Pore blocking is one of the main challenges with this mechanism [147]. In terms of flux, there are two types of flux: direct or surface flux $[142,149]$. The direct flux is defined as the gas molecules passing directly through the pore from one bulk phase to the other bulk phase on the permeate side of the graphene. The surface flux is defined as the crossing of a molecule to the other side of the graphene membrane after it has been adsorbed to the surface, a sort of slipping through the pore after its adsorbed on the surface. These types of flux have also been classified with a similar name: two-dimensional (2D) and three-dimensional (3D) flux. Similarly, the 3D diffusion is when the molecule finds the pore in the 3D space and 2D diffusion is when molecules are adsorbed and diffuse when they find the pore along the sheet.

Some gasses do not adsorb onto the graphene membrane so easily such as $\mathrm{He}$ and $\mathrm{H}_{2}$ (weak adsorption),[142,149] making their surface flux negligible. However, for the gasses that to have adsorption ability, such as $\mathrm{CH}_{4}$ and $\mathrm{N}_{2}, \mathrm{CO}_{2}$ (strong adsorption), their flux is appreciably affected. [142,149] For example, in a study of the separation of $\mathrm{H}_{2}$ and $\mathrm{N}_{2}$, the driving mechanism was stated to be the van der Waals forces. These forces led the $\mathrm{N}_{2}$ molecule to distribute on the graphene surface, causing the $\mathrm{N}_{2}$ to diffuse in a $2 \mathrm{D}$ manner, which led an increase in the $\mathrm{N}_{2}$ permeation, whereas the $\mathrm{H}_{2}$ has to go through 3D diffusion. These interactions are so impactful that despite $\mathrm{H}_{2}$ being smaller than $\mathrm{N}_{2}, \mathrm{~N}_{2}$ permeates in a more facile way [107] When a gas molecule is just bigger than the pore diameter, mechanisms such as diffusion, surface adsorption, and condensation of the molecules in the pores, are what govern the transport of the gas molecules through the membrane $[108,150]$. However, the principle of steric exclusion continues to apply and as the pore size increases, these different diffusions (2D vs 3D) become less significant and selectivity decreases. Table 1. lists selectivity ratios for gas separation through NPG membranes.

Table 1: Selectivity ratios for gas separation with NPG membranes.

\begin{tabular}{|c|c|c|c|c|c|}
\hline Gas & Selectivity Ratio & Type of Study & Pore Size & Graphene Type & Ref. \\
\hline \multirow{5}{*}{$\mathbf{H}_{2} / \mathbf{C H}_{4}$} & 1024 & MD & $2.8 \AA$ & Polyphenylene bilayer & {$[151]$} \\
\cline { 2 - 5 } & 1023 & MD, DTF & $2.5 \AA \times 3.8 \AA$ & Single sheet H-pore & {$[103]$} \\
\cline { 2 - 5 } & 108 & MD, DTF & $3.0 \AA \times 3.8 \AA$ & Single sheet N-pore & {$[103]$} \\
\cline { 2 - 6 } & 104 & Experimental & $\sim 3.4 \AA$ & Bilayer & {$[16]$} \\
\cline { 2 - 6 } & 8.75 & MD & $3.8165 \AA$ & Bilayer & Bilayer \\
\hline \multirow{2}{*}{$\mathbf{H}_{2} / \mathbf{C O}_{2}$} & 5 & Experimental & $\sim 4.9 \AA$ & {$[106]$} \\
\cline { 2 - 6 } & 1.7 & Experimental & $\sim 4.9 \AA$ & Bilayer \\
\hline
\end{tabular}




\begin{tabular}{|c|c|c|c|c|c|}
\hline \multirow{4}{*}{$\mathrm{CO}_{2} / \mathrm{N}_{2}$} & $100 \%$ (No CO2) & MD, DTF & Diameter $4.06 \AA$ & H-pore Single & [145] \\
\hline & $7 \times 103$ & Experimental & $\sim 3.4 \AA$ & Bilayer & [16] \\
\hline & 14.4 & MD & Diameter $0.99 \mathrm{~nm}$ & Porous graphene & [152] \\
\hline & 3.6 & Experimental & $\sim 4.9 \AA$ & Bilayer & [16] \\
\hline \multirow{3}{*}{$\mathrm{CO}_{2} / \mathrm{CH}_{4}$} & $9 \times 103$ & Experimental & $\sim 3.4 \AA$ & Bilayer & [16] \\
\hline & 9.9 & MD & Diameter $0.99 \mathrm{~nm}$ & Porous graphene & [152] \\
\hline & 1.7 & Experimental & $\sim 4.9 \AA$ & Bilayer & {$[16]$} \\
\hline \multirow{3}{*}{$\mathbf{H}_{2} / \mathbf{N}_{2}$} & 104 & Experimental & $\sim 3.4 \AA$ & Bilayer & [16] \\
\hline & 11 & Experimental & Kinetic diameter cutoff $4.9 \AA$ & Bilayer & [16] \\
\hline & 2.81 & MD & $3.8165 \mathrm{~A}$ & polyphenylene & [106] \\
\hline \multirow{2}{*}{$\mathrm{CH} 4 / \mathrm{H} 2 \mathrm{~S}$} & 8.01 & MD & & Charged carbon pore & [105] \\
\hline & 2.44 & MD & & Pristine pore & [105] \\
\hline \multirow{2}{*}{$\mathrm{N}_{2} / \mathrm{CH}_{4}$} & 1.3 & Experimental & $\sim 3.4 \AA$ & Bilayer & [16] \\
\hline & 0.5 & Experimental & $\sim 4.9 \AA$ & Bilayer & [16] \\
\hline $\mathbf{H}_{2} / \mathbf{A r}$ & $2 \times 1013$ & Experimental & $\sim 3.4 \AA$ & Bilayer & [16] \\
\hline $\mathrm{He} / \mathrm{Ar}$ & 3.75 & Experimental & $\sim<2.6 \AA$ Å diameter & Porous graphene & [6] \\
\hline $\mathrm{H}_{2} / \mathrm{SF}_{6}$ & $100 \%$ & Experimental & $\sim 4.9 \AA$ & Bilayer & {$[16]$} \\
\hline $\mathrm{He} / \mathrm{SF}_{6}$ & 8.4 & Experimental & $\sim<2.6 \AA \AA$ diameter & Porous graphene & [6] \\
\hline $\mathrm{CO}_{2} / \mathrm{SF}_{6}$ & $100 \%$ & Experimental & $\sim 4.9 \AA$ & Bilayer & {$[16]$} \\
\hline $\mathrm{CH}_{4} / \mathrm{SF}_{6}$ & $100 \%$ & Experimental & $\sim 4.9 \AA ̊$ & Bilayer & [16] \\
\hline $\mathbf{N}_{2} / \mathrm{SF}_{6}$ & $100 \%$ & Experimental & $\sim 4.9 \AA$ & Bilayer & {$[16]$} \\
\hline $\mathrm{CO}_{2} / \mathrm{Ar}$ & $5 \times 102$ & Experimental & $\sim 3.4 \AA$ & Bilayer & [16] \\
\hline $\mathrm{Ar} / \mathrm{N}_{2}$ & 5 & Experimental & $\sim 3.4 \AA$ & Bilayer & [16] \\
\hline $\mathrm{Ar} / \mathrm{CH}_{4}$ & 7 & Experimental & $\sim 3.4 \AA$ & Bilayer & [16] \\
\hline $\mathrm{He} / \mathrm{H}_{2}$ & 0.81 & Experimental & $\sim<2.6 \AA$ Å diameter & Porous graphene & [6] \\
\hline $\mathrm{He} / \mathrm{CO}_{2}$ & 4.18 & Experimental & $\sim<2.6$ Å diameter & Porous graphene & {$[6]$} \\
\hline $\mathrm{He} / \mathrm{O}_{2}$ & 3.4 & Experimental & $\sim<2.6 \AA \AA$ diameter & Porous graphene & [6] \\
\hline $\mathrm{He} / \mathrm{N}_{2}$ & 3.28 & Experimental & $\sim<2.6 \AA \AA$ diameter & Porous graphene & [6] \\
\hline $\mathrm{He} / \mathrm{CH}_{4}$ & 2.57 & Experimental & $\sim<2.6 \AA$ Å diameter & Porous graphene & [6] \\
\hline $\mathrm{CO}_{2} / \mathrm{O}_{2}$ & 9.5 & MD & $0.99 \mathrm{~nm}$ diameter & Porous graphene & [152] \\
\hline $\mathrm{H}_{2} / \mathrm{CO}$ & 2.6 & MD & $3.8165 \AA$ & Polyphenylene & [106] \\
\hline $\mathrm{H}_{2} / \mathrm{CO}_{2}$ & 3400 & Experimental & & Non porous- GO & [43] \\
\hline $\mathrm{CO}_{2} / \mathrm{N}_{2}$ & 382 & Experiment, MD & & GO membranes w/ ionic liquid & [41] \\
\hline $\mathbf{H}_{2} / \mathbf{N}_{2}$ & 900 & Experimental & & Filtrated GO membranes & [43] \\
\hline
\end{tabular}

\section{Transport Mechanisms Through Nano Channels}

Nano channels (NC) within graphene, are considered one of the mostpromising structures for water, gas, and ion transport $[109,111]$ . Basically, these channels exist between adjacent layers of graphene sheets in multilayer graphene, or graphene oxide, and, if ideally designed, can provide highly efficient pathways for transport. Mass transport occurs through the nanocapillaries of NCs. NCs designs vary [2,151-154] but are generally composed of stacked graphene sheets or a graphene architecture that generates nanocapillaries, or channels, resulting in a single-file line molecular sieving. In fact, NCs resemble naturally occurring channels in aquaporins or other proteins channels. These natural channels possess highly efficient naturally excluding ion techniques which work through either steric exclusion or electrostatic interactions. [153,155,156]
Akin to the natural occurring channels, NCs enables separation via size exclusion or charge repulsion $[32,157]$ The details of transport mechanisms through NCs will be discussed in the following section.

\section{Ion Transport Through Nano Channels}

Ion transport through graphene NCs happen in two main mechanisms i) steric exclusion and ii) Donnan-type interactions, (Figure 4) e-f. In this section we will cover the mechanisms and the parameters that induce them.

\section{Steric Exclusion in Graphene Nano Channels:}

Steric exclusion is omnipresent in dictating ion transport through NCs based membranes. In principle, the larger an ion is, the more difficult it becomes to enter the channels of the membrane and permeate through it. This is because the larger an 
ion, the higher the energy barrier needed to be overcome to enter the channel becomes. It is difficult for ions to squeeze through the channels by deforming their hydration shells,[154] allowing the smaller ions, or water molecules to travel through the membrane easier, inducing selectivity. In general, ions with stronger hydration shells are expected to have larger barriers for entry into NCs and exponentially smaller permeation rates. In a notable example for selectivity through steric exclusion, Joshi et al.[30] did not find correlation to ion charge, but just the ion's hydrated radius. For example, a highly negative arsenite ion $\left(\mathrm{AsO}^{43-)}\right.$ was filtered at the same rate of either $\mathrm{Na}+$ or $\mathrm{Cl}$-. In this experimental study, the solutes that had a hydrated radius larger than $4.5 \AA$, were blocked from the membranes, however, smaller ions permeated at rates thousands of times faster than predicted for a simple diffusion model, which can be explained by high capillary-like pressures on the small ions when they are inside the channels. Ion transport through graphene NCs is highly affected by the interlayer distance between adjacent layers. As such the most important parameter for this mechanism is the interlayer distance of the membrane's nanocapillaries. [57] For example, when an experimental GO membrane had an interlayer distance of $0.8 \mathrm{~nm}$, water permeation occurred, but this was not enough clearance for hydrated ions to permeate[48]. Ions in the nanocapillaries diffuse in a parallel manner to the graphene or GO sheets [109]. Depending on the application, the interlayer distance should be accommodated to fit the specific purpose. For example in desalination, the spacing of a GO membrane should not be much greater than the radius of hydrated ions such as hydrated $\mathrm{Na}+[157]$. Furthermore, in a recent study, the authors demonstrated a steric exclusion-based selectivity of $97 \%$ rejection for $\mathrm{NaCl}$ due to the interlayer spacing ( 9.8 to $6.4 \AA$ ) being smaller than the diameters of the ions and presenting an energy barrier at their entrance[45]. The energy barriers are a consequence of the interlayer spacing. They reported that by decreasing the interlayer spacing from 9.8 to $7.9 \AA$, the energy barrier increases from $20 \pm 2 \mathrm{kJmol}^{-1}$ to $72 \pm 7$ $\mathrm{kJmol}^{-1}$.

The interlayer spacing through which mass will travel can vary greatly. For example, this spacing is not always the random channels that are created when graphene sheets are randomly stacked upon each other but can be controlled by creating straight singlefile mass transport throughout the membrane thickness, (Figure 4) $[45,153,154]$ Gopinadha et al. [153] reports an extreme steric exclusion dominating mechanism. They fabricated a graphenebased water channels with $3.4 \AA$ interlayer spacing through van der Waals assembly. It was effective in completely removing $\mathrm{K}+$ and $\mathrm{Cl}$ - ions, which are double the magnitude of the height of the capillaries, but did not restrict water flow, as water molecules are $2.8 \AA$ in size. Abraham et al.[45]'s approach to control interlayer spacing was through encapsulating GO sheets with epoxy when they has water molecules as spacers, making the interlayer distance just enough for water. Their composite demonstrated $97 \%$ rejection of $\mathrm{NaCl}$. This steric exclusion happened because of the capillary size was smaller than the hydrated ion diameters, which as the capillary size decreased, the energy barrier for these ions increased, allowed water molecules through, as they were affected significantly less by the size barrier.

Control of the interlayer spacing is an ongoing challenge. Especially for applications such as water desalination, the GO membranes are exposed to aqueous solutions, making them vulnerable to swelling. Swelling in GO and NCs frameworks can be defined as the intercalation of water molecules between the sheets and can be as big as 2-3 layer of water molecules $\AA$ [45] or larger [48] . This issue has been addressed by for example incorporating graphene flakes into GO laminates[45]. The composites showed significantly less swelling than the GO laminates (difference of 4 $\AA$ ). This is due to the graphene's hydrophobicity which limits water intake. Interestingly, an ion or a molecule traveling through a GO membrane does not consistently experience the same environment throughout all the channel lengths. Owing to its oxygen functional groups, GO favors singular ion steric exclusion mechanisms that greatly differ from NPG steric mechanisms [47,48]. The oxygen functional groups in GO membranes have the tendency of clustering together, leaving small non-oxidized graphene nanocapillary segments, which produce water flow with less friction. In this region where there is less accumulation of the functional groups, the water molecules flow easier due to the reduced friction, but during the oxidized regions, the molecules have less mobility due to the interaction with the hydrogen and the functional groups [48]. In general, smaller ions can more easily go through nanocapillaries $[33,48,117]$. The steric exclusion mechanism is truly present in any GO-NC membrane because the nanocapillaries fully enclose the ion throughout its path in the membrane, and different from NPG membranes the mass that travels through it must cross a large membrane thickness than perhaps a single graphene sheet NPG membrane has. In studying GO membrane structure, it is impossible to escape the effects of the functional groups. Despite the steric dependability for ionic sieving, the importance of Donnan-type interactions must be taken into the account in terms of design and functionality. The ions and functional groups that are added to the membrane, as well as the sieved-ion characteristics must both be considered. An example of this interaction is seen in a study by P. Sun and coworkers. They reported that electrostatic attractions and chemical interactions, e.g. the Donnan-type interactions, between these functional groups and hydrated ions are responsible for the selective penetration properties of GO membranes. The radius of the hydrated ions which were sieved was $\mathrm{Mn}^{2+}>\mathrm{Cd}^{2+}>\mathrm{Cu}^{2+}>\mathrm{Na}^{+}$, yet the selectivity remained $\mathrm{Na}^{+}>\mathrm{Mn}^{2+}>\mathrm{Cd}^{2+}>\mathrm{Cu}^{2+}$, suggesting the selectivity had a stronger derivation from the electrostatic interactions. The Donnan-type mechanism can be exploited when designing GO based membranes, specifically through controlling the interlayer thickness. Interlayer spacing controlling methods are commonly done by adding functional groups between the GO layers and through this bringing the layers together or serving as a kind of spacer to increase the height. This has been done through covalent cross linking, organic compounds and diamine monomers. L. Chen et al.[47] used $\mathrm{K}+, \mathrm{Na}+\mathrm{Ca}+\mathrm{Li}+$ or $\mathrm{Mg}+$ ions between GO sheets to bring precision on the interlayer spacing down to one angstrom. 
This addition of cations between the nanocapillaries by GO, can with precision filter different types of larger positives ions (e.g., interlinking with $\mathrm{Na}+$ can exclude $\mathrm{K}+\mathrm{Ca}^{2+}$ and $\mathrm{Mg}^{2+}$ ).

This is defined by cation- $\pi$ interactions. The mechanism that results is defined by the cations (ex, $\mathrm{Na}+$ ) moving between the two GO sheets to a position near the bottom GO sheet and adsorbing to the regions where oxidized groups and aromatics rings coexist, thereby allowing less ions through [32] had GO functionalized with hydroxyl and epoxy groups on the carbon plane $(10 \%$ and $9 \%$ to carbon atoms respectively) and carboxyl groups on the edge of the sheet. Using $\mathrm{MgCl}_{2}$ and $\mathrm{Na}_{2} \mathrm{SO}_{4}$ as representative divalent and monovalent salts, pristine $\mathrm{GO}$ had low $\mathrm{MgCl}_{2}$ rejection (10\%) because it has interlayer spacing up to $7 \mathrm{~nm}$. And it had $40 \%$ rejection of $\mathrm{Na}_{2} \mathrm{SO}_{4}$ because the membranes swelled way less $(2 \mathrm{~nm})$. The cross linking brought both salt rejection to $70 \%$. After reducing the cross-linked sheet, then the water flux increased by $150 \%$ to $12-15$ LMH bar-1. This is probably because removing the oxygen groups decreased the resistance to water transport within the GO channels. Interlayer distance in graphene NCs can also affect the performance of graphene-base supercapacitors. As discussed earlier, porous graphene store charge physically in the pores. Recently,[158] it was reported that optimal performance of these pores is highly affected by the interlayer distance. Optimal utilization of graphene pores is also directly related to the size of electrolyte ions. Thus, interlayer distance needs to be precisely adjusted to ensure optimal utilization of the pores and thus maximum energy storage performance.

\section{Gas Transport Through Graphene Oxide Nano Channels}

GO membranes have been used widely for gas separation. Again, stacking of the GO layers leads to the mass transport being through the nanocapillaries or the channels that are created between and alongside the stacked GO sheets [159]. As discussed earlier, the interlayer distance between the channels is the main criteria in determining selectivity. The interlayer distance is what defines the height of the nano channels created as the nanocapillaries, which can be made to be wide enough to allows gas molecules through them, but also narrow as necessary to sterically exclude the bigger molecules to bring selectivity. Additionally, the oxygen groups aid the GO in having a larger interlayer thickness but when other functional groups such as hydroxyl and carboxyl groups are introduced between the graphene sheets, the carboxyl group brings a repelling electrostatic interaction on the sheets, prevent their aggregation $[42,49]$. Humidity also plays an important role in the interlayer thickness. demonstrated that dry GO hinders even He gas, but when exposed to humidity, He could permeate because of the absorbing of the water molecules on the oxide functional groups, increasing the interlayer thickness.

\section{Water Transport Through Graphene Nanochannelss}

Graphene NCs provide ideal pathways for water treatment technologies. The structural characteristics of the NCs such as hydrophilicity level, interlayer distance and functional groups play critical roles in the overall efficiency of water transport process. A successful approach by [49] demonstrated this experimentally. The group showed that GO membranes can have an interlayer layer of $\sim 10 \AA$, which is enough to completely block out liquids, vapors and gases from penetration, however it allows water through. This is because of the steric induced selectivity, where only a monolayer of water, in single file line was allowed through the channels of the membrane. The interlayer spacing of the membrane channels determines the ease of the water molecules for permeating, thus bringing selectivity by steric exclusion [2,160]. Additionally, an interesting trend of fast water permeation has been seen in GO membranes. The ultra-fast water permeation through the GO laminates occurs due to their nanocapillaries bringing low friction to water molecules. The high water permeation that is seen as a trend in GO membranes can be explained by the advantageous slip flow that occurs along the channels [2,49] However, due to the GO's functional groups, the interlayer spacing must be carefully considered as environmental factors can affect its performance. For example the effect of wetting the graphene sheets causes the interlayer spacing to go from 0.9 to $1.3 \mathrm{~nm}$ from humid air to water, bringing rapid permeation of mono and di valent ions [30] This means that there is instability in water. Stability can be achieved by decreasing the interlayer spacing by removing some oxygen functional groups and also by either thermal or chemical reduction $[2,36,111]$. Recently, electric field was also used to increase water transport through NCs [161]. In this case, when the electric field around conductive filaments in the membrane was created, the water molecules become ionizes within the membrane, impeding the water molecule transport. This approach is precise, controllable and had incredible potential for a large-scale production. It has also been found that positive charges at the entrance of channels boost water transport while negative charges do not, as well as a correlation between the hydrogen bonding sites within water channels and the single channel permeability and the Gibbs activation energy for each channel [160].

\section{Conclusion}

Owing to its unique structural and chemical properties, graphene membrane offers many opportunities for mass transports. While graphene membrane has been used successfully for various application e.g., gas separation, supercapacitors and water desalination, its full potentials is yet to be explored. The most astonishing feature about graphene-based membrane-assisted technologies is that the mass transport can be controlled effectively via physical or chemical modification of graphene nanopores or nano channels. Optimal ion transport is the key for various energy storage and conversion systems such as fuel cell or supercapacitors. This optimization can be achieved by designing highly effective ion transport pathways such as nanochannels or pores with optimal density and sizes. For example, the size of graphene pores needs to be adjusted to the size of electrolyte ions. For applications such as water purification, increased selectivity can lead to significant performance improvement while retaining energy and mass savings. Selectivity can be tuned by adjusting the structural characteristics during the synthesis process, or during the transport process through voltage tuning, electric field implementation, 
and functionalization of pore edges, among others. Additionally, membrane selectivity is highly affected by the dehydration process e.g., ion dehydration. Studies on graphene membranes have been largely done in both computational (molecular dynamics, density functional theory, etc.) and experimental studies, however most of the MD studies performed under ideal conditions. MD simulations should be done with more realistic environments. For example, when studying graphene membranes often single pores are studied at a time. Moving forward, it is important to study, both through simulations and experiments, larger pore densities, rather than isolated pores, to bring real insight into how the translation of these membranes will be to real world applications.

While studies on a single pore are valuable to delineate fundamental science, the road to applicable knowledge is hindered by not studying larger scale systems with a high pore density. Pore density plays a big role in the efficiency of the membranes. Additionally, a significant problem faced with graphene membranes is difficulty of scaling same- sized characteristics. Apart from making large area monolayer NPG graphene, there must be uniformity in pores present. The precision of pore length is vital as unoptimized pores sizes largely decrease selectivity. Further, given that nano channels showed great potentials for water transport, it is highly anticipated that similar nano channels might be the best solution for selective mass transport. Graphene NCs can be used for both gas and ion transport. Finally, while graphene membrane's advantages have been successfully exploited for some applications such as water desalination, its huge potentials for multiples applications, e.g. as an ionomer in fuel cell, deserves further explorations.

\section{Conflicts of Interest}

The authors declare no conflict of interest

\section{References}

1. Baker RW (2012) Membrane Technology and Applications, Third Edition Membrane Technology and Applications, John Wiley \& Sons.

2. Werber JR, Osuji CO, M (2016) Elimelech Materials for next-generation desalination and water purification membranes. Nat Rev Mater 1.

3. Chabi S, Papadantonakis KM, Lewis NS, Freund MS (2017) Membranes for artificial photosynthesis Energy Environ Sci 10: 1320.

4. Jang D, Idrobo JC, Laoui T, Karnik R (2017) Water and Solute Transport Governed by Tunable Pore Size Distributions in Nanoporous Graphene Membranes. ACS Nano 11: 10042.

5. Russo CJ, Golovchenko JA (2012) Atom-by-atom nucleation and growth of graphene nanopores Proc. Natl. Acad. Sci. U. S. A. 109: 5953.

6. Boutilier MSH, Jang D, Idrobo JC, Kidambi PR, Hadjiconstantinou NG, et al. (2017) Molecular Sieving Across Centimeter-Scale Single-Layer Nanoporous Graphene Membranes. ACS Nano 11: 5726.

7. Celebi K, Buchheim J, Wyss RM, Droudian A, Gasser P, et al. (2014) Ultimate Permeation Across Atomically Thin Porous Graphene. Science (80)344: 289.

8. O’Hern SC, Boutilier MSH, Idrobo JC, Song Y, Kong J, et al. (2014) Selective ionic transport through tunable subnanometer pores in single-layer graphene membranes. Nano Lett $14: 1234$.
9. 'Hern SCO, Jang D, Bose S, Idrobo JC, Song Y, et al. (2015) Nanofiltration across Defect-Sealed Nanoporous Monolayer Graphene. Nano Lett 15: 3254.

10. Ghosh M, Jorissen KFA, Wood JA, Lammertink RGH (2018) Ion Transport through Perforated Graphene J. Phys. Chem. Lett 9: 6339.

11. Schneider GF, Xu Q Hage S, Luik S (2013) Tailoring the hydrophobicity of graphene for its use as nanopores for DNA translocation. Nat. Commun 2013 4: 1 .

12.Song B, Schneider GF, Xu Q, Pandraud G, Dekker C, et al. (2011) Characterization of the cleaning process on a transferred graphene Nano Lett 11: 2247.

13. Schneider GF, Kowalczyk SW, Calado VE, Pandraud G, Zandbergen HW, et al. (2010) Wedging Transfer of Nanostructures. L M Nano Lett 10: 3163.

14. Surwade SP, Smirnov SN, Vlassiouk IV, Unocic RR, Veith GM, et al. (2015) Water desalination using nanoporous single-layer graphene. Nat. Nanotechnol 10(5): 459-464.

15. Qi H, Li Z, Tao Y, Zhao W, Lin K, et al. (2018) Fabrication of sub-nanometer pores on graphene membrane for ion selective transport Nanoscale10: 5350.

16. Koenig SP, Wang L, Pellegrino J, Bunch JS (2012) Selective molecular sieving through porous graphene .Nat. Nanotechnol 7: 728.

17. Zhu Y, Murali S, Stoller MD, Ganesh KJ, Cai W ,et al. (2011) Carbon-Based Supercapacitors Produced by Activation of Graphene. Science (80) 332: 1537.

18. Ha J, SK Park, SH Yu, A Jin, B Jang, et al. (2013) A chemically activated graphene-encapsulated LiFePO4 composite for high-performance lithium ion batteries. Nanoscale 5: 8647

19. Zheng C, X Zhou, H Cao, G Wang, Z Liu (2014) J Power Sources 258: 290.

20. Liu S, W Peng, H Sun, S Wang (2014) Physical and chemical activation of reduced graphene oxide for enhanced adsorption and catalytic oxidation. Nanoscale 6: 766.

21. Wang S, H Sun, HM Ang, MO Tadé (2013) Chem. Eng. J 226 : 336.

22. Rollings RC, AT Kuan, JA Golovchenko (2016) on selectivity of graphene nanopores. Nat. Commun 7: 1.

23. Merchant CA, K Healy, M Wanunu, V Ray, N Peterman, et al. (2010) DNA Translocation through Graphene Nanopores. Nano Lett 10: 2915.

24. Venkatesan BM, D Estrada, S Banerjee, X Jin, V E Dorgan (2012) Stacked Graphene-Al203 Nanopore Sensors for Sensitive Detection of DNA and DNA-Protein Complexes. ACS Nano 6: 441.

25. Krasheninnikov AV, F Banhart (2007) Engineering of nanostructured carbon materials with electron or ion beams. Nat. Mater 6: 723.

26. Bell DC, MC Lemme, LA Stern, JR Williams, CM Marcus (2009) Precision Material Modification and Patterning with Helium Ions. Nanotechnology 20: 1 .

27. Yuan W, J Chen, G Shi, Mater (2014) Nanoporous graphene materials. Today 17: 77 .

28. Surwade SP, SN Smirnov, IV Vlassiouk, RR Unocic, GM Veith, (2015) Water desalination using nanoporous single-layer graphene. Nat. Nanotechnol 10: 459.

29. Zhang LL, X Zhao, MD Stoller, Y Zhu, H Ji (2012) Highly conductive and porous activated reduced graphene oxide films for high-power supercapacitors. Nano Lett 12: 1806.

30. Joshi RK, P Carbone, FC Wang, VG Kravets, Y Su, et al. (2014) Precise and Ultrafast Molecular Sieving Through Graphene Oxide Membranes. Science (80)343: 752 . 
31. Kumar R, K Scott (2012) Freestanding sulfonated graphene oxide paper: a new polymer electrolyte for polymer electrolyte fuel cells. Chem. Commun 48: 5584

32. Mi B, S Zheng, Q Tu (2018) 2D graphene oxide channel for water transport. Faraday Discuss 209: 329.

33. Zhang Y, S Zhang, T S Chung (2015) Nanometric Graphene Oxide Framework Membranes with Enhanced Heavy Metal Removal via Nanofiltration. Environ. Sci. Technol.

34. Chong JY, B Wang, K Li (2018) Water transport through graphene oxide membranes: the roles of driving forces. Chem. Commun 54: 2554

35. Han Y, Z Xu, C Gao (2013) Ultrathin Graphene Nanofiltration Membrane for Water Purification. Adv. Funct. Mater 23: 3693.

36. Su Y, V G Kravets, SL Wong, J Waters, AK Geim, (2014) Impermeable barrier films and protective coatings based on reduced graphene oxide. Nat. Commun.

37. Paneri A, Y Heo, G Ehlert, A Cottrill, H Sodano (2014) A selective electrocatalyst-based direct methanol fuel cell operated at high concentrations of methanol. J. Memb. Sci 467: 217.

38. Dikin DA, S Stankovich, E J Zimney, RD Piner, GHB Dommett ,et al. (2007) Graphene-based composite materials. Nature 442: 282-286.

39. Peng W, S Liu, H Sun, Y Yao, L Zhi, et al. (2013) Synthesis of porous reduced graphene oxide as metal-free carbon for adsorption and catalytic oxidation of organics in water. J Mater. Chem 1: 5854.

40. Luo Z, Y Lu, L A Somers, A T C Johnson (2009) Mosaic-like Monolayer of Graphene Oxide Sheets Decorated with Tetrabutylammonium Ions. J. Am. Chem. Soc 131: 898.

41. Ying W, J Cai, K Zhou, D Chen, Y Ying, et al. (2018) Preparing supported ionic liquids by continuous supercritical carbon dioxide adsorption method. ACS Nano12: 5385.

42. Shen J, G Liu, K Huang, Z Chu, W Jin, et al. (2016) Subnanometer TwoDimensional Graphene Oxide Channels for Ultrafast Gas Sieving. ACS Nano 10: 3398

43. Li H, Z Song, X Zhang, Y Huang, S Li, et al. (2013) Science (80) 342: 95

44. Liu G, H Ye, A Li, C Zhu, H Jiang, et al. (2016) Graphene Oxide- Inorganic Composite Membrane: A Review. Carbon N Y 110: 56.

45. Abraham J, KS Vasu, C D Williams, K Gopinadhan, Y Su,et al. (2017) Graphene Oxide Membranes for Tunable Ion Sieving in Acidic Radioactive Waste. Nat. Nanotechnol 12: 546.

46. Shen J, G Liu, K Huang, W Jin, KR Lee, et al. (2015) Membranes with Fast and Selective Gas-Transport Channels of Laminar Graphene Oxide for Efficient CO2 Capture. Angew. Chemie - Int. Ed. 54 : 578.

47. Chen L, G Shi, J Shen, B Peng, B Zhang, et al. (2017) Ion sieving in graphene oxide membranes via cationic control of interlayer spacing. Nature 550: 380-383.

48. Sun P, Zhu M, Wang K, Zhong M, Wei J, Wu D, et al. (2013) Selective ion penetration of graphene oxide membranes. ACS Nano 7: 428.

49. Nair RR, Wu HA, Jayaram PN, Grigorieva IV, Geim AK (2012) Unimpeded Permeation of Water Through Helium-Leak-Tight Graphene-Based Membranes. Science (80).

50. Yoo JJ, Balakrishnan K, Huang J, Meunier V, Sumpter BG, et al. Ultrathin planar graphene supercapacitors. (2011) Nano Lett.

51. Bai J, Zhong X, Jiang S, Huang Y, Duan X (2010) Nat. Nanotechnol. 5:190.

52. Liang X, Jung YS, Wu S, Ismach A, Olynick DL, at al (2010) Nano Lett.10: 2454 .
53. Hu S, Lozada-Hidalgo M, Wang FC, Mishchenko A, Schedin F, at al (2014) Nature 516: 227.

54. Chabi S, Peng C, Yang Z, Xia Y, Zhu Y (2015) RSC Adv. 3999.

55. X. Wang, Y. Zhang, C. Zhi, X. Wang, D. Tang, Y at al. (2013) Nat. Commun 2905.

56. Morelos-Gomez A, Cruz-Silva R, Muramatsu H, Ortiz-Medina J, Araki T (2017) Nat. Nanotechnol. 12:1083.

57. Hung WS, Tsou CH, De Guzman M, An QF, Liu YL, Zhang YM, Hu CC, Lee KR, Lai JY, (2014) Chem. Mater. 26(9).

58. Park J, Bazylewski P, Fanchini G (2016) Nanoscale 18.

59. Rajput A, Sharma PP, Yadav V, Kulshrestha V (2020) Int. J. Hydrogen Energy 45:16976.

60. Bayer T, Cunning BV, Selyanchyn R, Daio T, Nishihara M, (2016) Sci 508: 51.

61. Lin B, Yuan W, Xu F, Chen Q Zhu H (2018) Appl. Surf. Sci. 455-295.

62. Chakraborty U (2016) Appl. Energy 163:60.

63. Baik KD, Hong BK, Kim MS (2013) Renew. Energy 57-234.

64. Peighambardoust SJ, Rowshanzamir S, Amjadi M (2010) in Int. J. Hydrogen Energy.

65. Bayer T, Selyanchyn R, Fujikawa S, Sasaki K, Lyth SM, Memb J (2017) Sci. 541:347.

66. Yee RSL, Rozendal RA, Zhang K, Ladewig BP (2012) Chem. Eng. Res. Des. 90: 950.

67. Hu B, L. Miao L, Zhao Y, Lü C, Memb J (2017) Sci. 2017 530: 84.

68. Farooqui UR, Ahmad AL, Hamid NA (2018) Renew. Sustain. Energy Rev 82: 714 .

69. Dai P, Mo ZH, Xu RW, Zhang S, Lin X, (2016) RSC Adv 6: 52122.

70. Liu X, Chen X, Hu Y, Gong T, Li H, (2017) J. Electrochem. Soc.164:F433.

71. F. Chu, B. Lin, T. Feng, C. Wang, S. Zhang, (2015) Sci. 496:31.

72. Yang Q, Lin CX, Liu FH, Li L, Zhang QG at al (2018) Sci. 552:367.

73. Li Y, Shi S, Cao H, Zhao Z, Su ZC, at al (2018) Sci. 566: 44.

74. Chu JY, Lee KH, Kim AR, Yoo DJ, Memb J (2020) Sci. 611:118385.

75. Chu JY, Lee KH, Kim AR, Yoo DJ (2019) Compos. Part B Eng. 164: 324.

76. S Gao, H Xu, Z Fang, A Ouadah, H Chen (2018) Electrochim. Acta 283: 428.

77. Roelofs KS, Kampa A, Hirth T, Schiestel T, Appl J (2009) Polym. Sci. 111: 2998.

78. O Movil, L Frank, JA Staser, J (2015) Electrochem. Soc. 162: F419.

79. S Gahlot, PP Sharma, V Kulshrestha, PK Jha (2014) ACS Appl. Mater. Interfaces 6: 5595.

80. Z Yang, J Zhou, S Wang, J Hou, L. Wu T, et al. (2015) J. Mater. Chem. A 3: 15015.

81. MB McDonald, JP Bruce, K McEleney, MS Freund (2015) ChemSusChem

82. AT Najafabadi, E Gyenge (2014) Carbon NY 71: 58.

83. J Lu, JX Yang, J Wang, A Lim, S Wang, KP Loh (2009) ACS Nano 3: 2367.

84. X Zhang, J Li, Y Sun, Z Li, P Liu, Q Liu, et al. (2018) J. Guo, Electrochim. Acta 282: 626 .

85. IR Vazques, S Chabi (2020) in Proc 2020 ASEE Gulf-Southwest Annu. Conf., 2020. 
86. Z Luo, Q Xiao, G Lei, Z Li, C Tang (2016) Carbon N. Y, 98: 373.

87. J Wang, Wang J, H Liu, Z You, Z Li, et al. (2020) Adv Funct Mater 2007397.

88. SC Park, TH Lee, GH Moon, BS Kim, JM Roh, et al. (2019) ACS Appl. Energy Mater 2: 4590.

89. S Chabi, C Peng, D Hu, Y Zhu (2014) Adv. Mater. 26: 2440.

90. V Strauss, K Marsh, MD Kowal, M El-Kady, RB Kaner (2018) Adv. Mater 30: 1704449 .

91. TH Tight (2012) Science 335: 442.

92. JQ Huang, TZ Zhuang, Q Zhang, HJ Peng, CM Chen, et al. (2015) ACS Nano 9: 3002.

93. J Eliasson (2015) Nature 517:6.

94. Group Water Resources, Charting Our Water Future: Economic Frameworks to Inform Decision-Making, 2009.

95. KS Spiegler, YM El-Sayed (2001) Desalination 134: 109.

96. C Lee, X Wei, JW Kysar (2008) J. Hone, Science 321: 385.

97. D Cohen-Tanugi, JC Grossman (2012) Nano Lett, 12, 3602.

98. JR Klaehn, CJ Orme, ES Peterson, FF Stewart, JM Urban-Klaehn (2011) Inorganic Polymeric and Composite Membranes. Membr. Sci. Technol.

99. AE Yaroshchuk (2001) Sep. Purif. Technol., 22-23: 143.

100. JR Werber, A Deshmukh, M Elimelech (2016) Environ. Sci. Technol. Lett. 3: 112 .

101. Novoselov KS, Geim AK, Morozov SV, Jiang D, Katsnelson MI, et al (2005) Nature Two-dimensional gas of massless Dirac fermions in graphene. 438: 197-200.

102. C Moreno, M Vilas varela, B Kretz, A Garcia lekue, MV Costache, et al. (2018) Bottom-up synthesis of multifunctional nanoporous graphene Science 360(6385):199-203.

103. DE Jiang, VR Cooper, S Daiz (2009) Porous graphene as the ultimate membrane for gas separation. Nano Lett 9(12): 4019-424.

104. Y Liu, X Chen (2014) J Appl Phys pp.115.

105. G Lei, C Liu, H Xie, F Song (2014) Chem Phys Lett 599: pp.127.

106. Y Tao, Q Xue, Z Liu, M Shan, C Ling, et al. (2014) Tunable Hydrogen Separation in Porous Graphene Membrane: First-Principle and Molecular Dynamic Simulation. ACS Appl Mater Interfaces 6: 8048 $-8058$.

107. H Du, J Li, J Zhang, G Su, X Li, et al. (2011) Separation of Hydrogen and Nitrogen Gases with Porous Graphene Membrane. J Phys Chem C 115: 23261-23266.

108. L Wang, MSH Boutilier, PR Kidambi, D Jang, NG Hadjiconstantinou, et al. (20107) Fundamental transport mechanisms, fabrication and potential applications of nanoporous atomically thin membranes. Nanotechnol 12: 509-522.

109. S Homaeigohar, M Elbahri (2017) Graphene membranes for water desalination. NPG Asia Mater 9 (8): e427.

110. Y Zhang, TS Chung (2017). Curr Opin Chem Eng 16: p.9.

111. P Sun, K Wang, H Zhu (2016) Employing Lead Thiocyanate Additive to Reduce the Hysteresis and Boost the Fill Factor of Planar Perovskite Solar Cells. Adv Mater 28: 5214-5221.

112. Z Song, Z Xu (2015) Sealing the Pores of PEO Coating with MgAl Layered Double Hydroxide: Enhanced Corrosion Resistance, Cytocompatibility and Drug Delivery Ability. Sci Rep 5: p.1.

113. K Sint, BY Wang, P Kral (2009) Selective Ion Passage through Functionalized Graphene Nanopores. J Am Chem Soc 131: pp.9600.
114. S Sahu, M Di Ventra, M Zwolak (2017) Dehydration as a Universal Mechanism for Ion Selectivity in Graphene and Other Atomically Thin Pores. Nano Lett 17: 4719 -4724.

115. D Konatham, J Yu, TA Ho, A Striolo (2013) Simulation Insights for Graphene-Based Water Desalination Membranes. Langmuir 29: 11884-11897.

116. Q Shi, Z He, KM Gupta, Y Wang, R Lu (2017) J Mater Sci 52: pp.173.

117. C Fang, H Wu, SY Lee, RL Mahajan, R Qiao (2018) Carbon NY 136: pp.262.

118. R Epsztein, E Shaulsky, M Qin, M Elimelech (2019) Towards singlespecies selectivity of membranes with subnanometre pores. J Memb Sci 580: pp.316.

119. Y Fu, S Su, N Zhang, Y Wang, X Guo, et al. (2020) A Synergistic Relationship between Polycaprolactone and Natural Polymers Enhances the Physical Properties and Biological Activity of Scaffolds. ACS Appl Mater Interfaces 12: 13587-13597.

120. A Seidel, JJ Waypa, M Elimelech (2001) Role of Charge (Donnan) Exclusion in Removal of Arsenic from Water by a Negatively Charged Porous Nanofiltration Membrane. Environ Eng Sci 18: pp.105.

121. J Azamat, A Khataee, SW Joo (2015) Separation of cyanide from an aqueous solution using armchair silicon carbide nanotubes: insights from molecular dynamics simulations. Chem Eng Sci 127: pp.285.

122. JG Gai, XL Gong (2014) An ultrafast water transport forward osmosis membrane: porous graphene. J Mater Chem A 2: pp.425.

123. Z He, J Zhou, X Lu, B Corry (2013) Bioinspired Graphene Nanopores with Voltage-Tunable Ion Selectivity for $\mathrm{Na}+$ and K+. ACS Nano 7: $10148-10157$.

124. Y Kang, Z Zhang, H Shi, J Zhang, L Liang, et al.(2014) Na+ and K+ ion selectivity by size-controlled biomimetic graphene nanopores. Nanoscale 6: pp.10666.

125. A Lohrasebi, S Rikhtehgaran (2018) Ion separation and water purification by applying external electric field on porous graphene membrane. Nano Res 11: pp.2229.

126. S Zhao, J Xue, W Kang (2013) Ion selection of charge-modified large nanopores in a graphene sheet. J Chem Phys 139: pp.114702.

127. DJ Cole, PK Ang, KP Loh (2011) Ion Adsorption at the Graphene/ Electrolyte Interface. J Phys Chem Lett 14: 1799-1803.

128. ME Suk, NR Aluru (2014) Ion transport in sub-5-nm graphene nanopores. J Chem Phys 140.

129. D Cohen Tanugi, LC Lin, JC Grossman (2016) Multilayer Nanoporous Graphene Membranes for Water Desalination. acs nanolett 16(2): 1027-1033.

130. Y Wang, Z He, KM Gupta, Q Shi, R Lu (2017) Thermo-osmosis in hydrophilic nanochannels: mechanism and size effect. Carbon NY 116: pp.120.

131. Cohen-Tanugi D, Grossman JC (2014) Water permeability of nanoporous graphene at realistic pressures for reverse osmosis desalination. J Chem Phys 141.

132. Nguyen CT, Beskok A (2019) Charged nanoporous graphene membranes for water desalination. Phys. Chem. Chem. Phys. 21: 9483.

133. Wang L, Williams CM, Boutilier MSH, Kidambi PR, Karnik R (2017) Single-Layer Graphene Membranes Withstand Ultrahigh Applied Pressure. Nano Lett 17(5).

134. Walker MI, Braeuninger-Weimer P,. Weatherup RS, Hofmann S, Keyser UF (2015) Measuring the proton selectivity of graphene membranes. Appl. Phys. Lett. 107(21). 
135. Achtyl JL. Unocic RR, Xu L, Cai Y, Raju M, et al. (2015) Aqueous proton transfer across single-layer graphene. Nat. Commun. 6: 1.

136. Poltavsky I, Zheng L, Mortazavi M, Tkatchenko A (2018) Quantum tunneling of thermal protons through pristine graphene. J. Chem. Phys. 148.

137. Lozada-Hidalgo M, Hu S, Marshall O, Mishchenko A, Grigorenko AN et al. (2016) Sieving hydrogen isotopes through two-dimensional crystals. Science (80)351: 68.

138. Gai JG, Gong XL. Wang WW, Zhang X, Kang WL (2014) J. Mater. Chem. A 2: 4023 .

139. Suk ME, Aluru NR (2013) Molecular and continuum hydrodynamics in graphene nanopores. RSC Adv. 3: 9365.

140. Zhu C, Li H, Meng S (2014) Transport behavior of water molecules through two-dimensional nanopores. J. Chem. Phys. 141.

141. Garnier L, Szymczyk A, Malfreyt P, Ghoufi A (2016) Physics behind Water Transport through Nanoporous Boron Nitride and Graphene. J. Phys. Chem. Lett 7: 3371.

142. Sun C, Boutilier MSH, Au H, Poesio P, Bai B, et al. (2014) Mechanisms of Molecular Permeation through Nanoporous Graphene Membranes. 30: 675.

143. Wu T, Xue Q, Ling C, Shan M, Liu Z et al. (2014) Fluorine-Modified Porous Graphene as Membrane for CO2/N2 Separation: Molecular Dynamic and First-Principles Simulations. J. Phys. Chem. C 118.

144. Liu H, Dai S, Jiang DE (2013) Nanoscale 5: 9984.

145. Wang Y, Yang Q, Li J, Yang J, Zhong C (2016) High anisotropy of fully hydrogenated borophene. Phys. Chem. Chem. Phys. 18: 8352.

146. Shan M, Xue Q Jing N, Ling C, Zhang $T$ et al. (2012) Influence of chemical functionalization on the $\mathrm{CO} / \mathrm{N} 2$ separation performance of porous graphene membranes. Nanoscale 17.

147. Drahushuk LW, Strano MS (2012) CO2 Adsorption on Carbon Models of Organic Constituents of Gas Shale and Coal. Langmuir 28: 16671.

148. Liu Y, Wilcox J (2012) CO2 Adsorption on Carbon Models of Organic Constituents of Gas Shale and Coal. Environ. Sci. Technol. 45.
149. Wang S, Tian Z, Dai S, Jiang DE (2018) Effects of Surface Heterogeneity on the Adsorption of $\mathrm{CO} 2$ in Microporous Carbons. Nanoscale 10: 14660.

150. Robeson LM, Memb J (2008) Sci. 320: 390.

151. Huang C, Wu H, Deng K, Tang W, Kan E (2014) Improving the photocatalytic activity and anti-photocorrosion of semiconductor $\mathrm{ZnO}$ by coupling with versatile carbon. Phys. Chem. Chem. Phys. 16: 25755.

152. Lee J, Aluru NR, Memb J (2013) Water-solubility-driven separation of gases using graphene membrane. Sci. 428: 546.

153. Gopinadhan K, Hu S, Esfandiar A, Lozada-Hidalgo M, Wang FC, et al. (2019) Complete steric exclusion of ions and proton transport through confined monolayer water. Science (80) 363: 145.

154. Radha B, Esfandiar A, Wang FC, Rooney AP, Gopinadhan K, et al . (2016) 538: 222 .

155. Shen YX, Song WC, Ryan Barden D, Ren T, Lang C, et al. (2018 Nat. Commun 9: 1 .

156. Jain T, Rasera BC, Guerrero RJS, Boutilier MSH, O’Hern SC, et al. (2015) Nanopores in two-dimensional materials: accurate fabrication. Materials Horizon 10: 1053.

157. Mi B (2014) Materials science. Graphene oxide membranes for ionic and molecular sieving. Science 343: 740.

158. Li Z, Gadipelli S, Li H. Howard CA, Brett DJL, et al. (2020) 2D graphene oxide liquid crystal for realworld applications: Energy, environment, and antimicrobial. Nat. Energy 5: 160.

159. Yoo BM, Shin JE, Lee HD, Park HB (2017) Graphene and graphene oxide membranes for gas separation applications. Curr. Opin. Chem. Eng 16: 39-47.

160. Barboiu M, Kumar M, Baaden M, Gale PA, Hinds BJ (2019) Highlights from the Faraday Discussion on Artificial Water Channels, Glasgow, UK. Chem. Commun. 55: 3853.

161. Zhou KG, Vasu KS, Cherian CT, Neek-Amal M, Zhang JC, et al. (2018) Electrically controlled water permeation through graphene oxide membranes. Nature 559: 236-240.

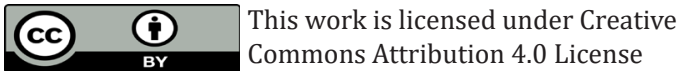

To Submit Your Article Click Here:

Submit Article

DOI: $10.32474 /$ MAMS.2021.04.000194

Citation: Irma Rocio Vazquez, Sakineh Chabi*. Graphene Membranes: Transport Properties and Energy Applications. Mod App Matrl Sci 4(4)2021. MAMS.MS.ID.000194. DOI: 10.32474/MAMS.2021.04.000194

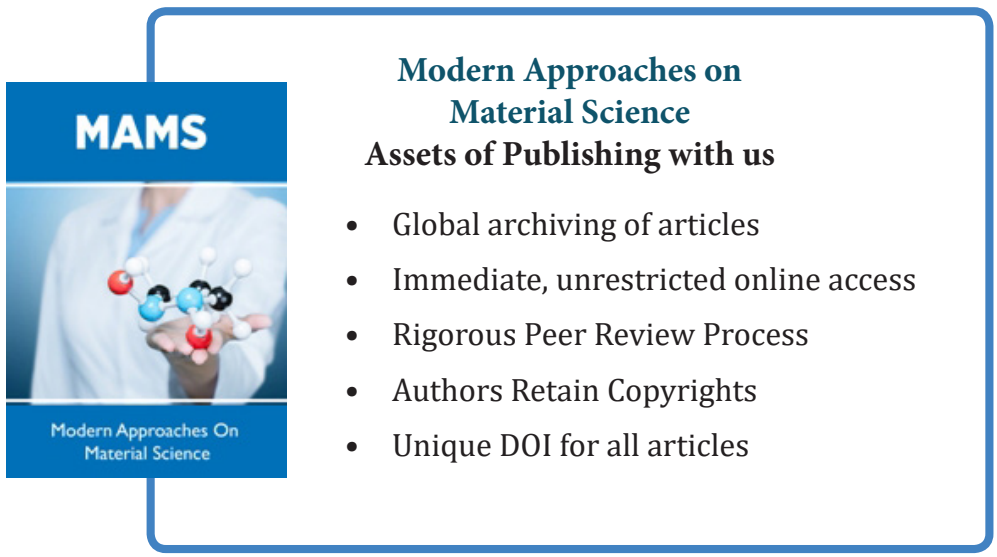

